\title{
CAUSAL AND STABLE INPUT/OUTPUT STRUCTURES ON MULTIDIMENSIONAL BEHAVIORS*
}

\author{
J. WOOD ${ }^{\dagger}$, V. R. SULE ${ }^{\ddagger}$ AND E. ROGERS ${ }^{\dagger}$
}

\begin{abstract}
In this work we study multidimensional (nD) linear differential behaviors with a distinguished independent variable, called "time." We define in a natural way causality and stability of input/output structures with respect to this distinguished direction. We make an extension of some results in the theory of partial differential equations, demonstrating that causality is equivalent to a property of the transfer matrix which is essentially hyperbolicity of the $P^{c}$ operator defining the behavior $\left(\mathcal{B}^{c}\right)_{0, y}$. We also quote results which in effect characterize time-autonomy for the general systems case.

Stability is likewise characterized by a property of the transfer matrix. We prove this result for the $2 \mathrm{D}$ case and for the case of a single equation; for the general case it requires solution of an open problem concerning the geometry of a particular set in $\mathbb{C}^{n}$. In order to characterize input/output stability we also develop new results on inclusions of kernels, freeness of variables, and closure with respect to $\mathcal{S}, \mathcal{S}^{\prime}$ and associated spaces, which are of independent interest. We also discuss stability of autonomous behaviors, which we believe to be governed by a corresponding condition.
\end{abstract}

Key words. stability, causality, partial differential equations, multidimensional systems, behavioral approach, hyperbolic systems, input/output structures, time-autonomy.

AMS subject classifications. 13C05, 13C12, 35B37, 93A99, 93B25, 93B55

DOI. $10.1137 /$ S0363012903429979

1. Introduction. In this paper we are concerned with questions of causality and stability for systems defined by PDEs. We consider these problems in the framework of multidimensional $(\mathrm{nD})$ behaviors (note that this is quite distinct from the infinitedimensional systems framework of, e.g., [2]). To date, the theory of $\mathrm{nD}$ behaviors has almost entirely considered the independent variables on an equal footing. However, in an apparent majority of applications, particularly in the case of systems given by PDEs, one of the independent variables, "time," is distinguished and plays a special role. Recent work by Sasane $[26,24,25]$ attempts to develop $\mathrm{nD}$ behavioral theory in this less symmetrical and more applicable situation.

This consideration is particularly significant when we discuss a concept such as stability, which is naturally associated with the passage of "time." Stability of course may be divided into two concepts: stability with respect to initial conditions (i.e., stability of an autonomous behavior) and input/output stability. The current work was motivated by consideration of the first concept but has led only to a (partial) characterization of the second!

We therefore begin our main exposition with a discussion of stability of autonomous behaviors in section 3. Our argument is motivated by the rough principle that a behavior should be classified as "unstable" only when it contains trajectories which are unstable (in whatever sense) but for which the corresponding initial conditions (whatever we may mean by these) are, nevertheless, (in some sense) stable. The two-dimensional (2D) discrete definition of stability by Valcher [33] applies this

*Received by the editors June 23, 2003; accepted for publication (in revised form) April 26, 2004; published electronically January 27, 2005.

http://www.siam.org/journals/sicon/43-4/42997.html

${ }^{\dagger}$ School of Electronics and Computer Science, University of Southampton, Southampton, SO17, 1BJ, UK (etar@ecs.soton.ac.uk).

$\ddagger$ Department of Electrical Engineering, Indian Institute of Technology Bombay, Powai, Mumbai, 400 076, India (vrs@ee.iitb.ac.in). 
principle. Unfortunately, for PDEs, "initial conditions" is a much harder concept to understand and work with. Section 3, however, proposes a possible condition for stability in this sense, which we call the characteristic variety (CV) condition. Roughly, the idea behind this condition is to classify trajectories as unstable only if they are both "blowing up" in the time direction and "physically reasonable" in the spatial direction. Section 3.1 brings hyperbolic systems into the discussion, since hyperbolicity is for certain important classes of systems a consequence of the CV condition. One particular property of hyperbolic systems is that they are time-autonomous as defined in [25]; we quote a result from the PDE literature which effectively characterizes this property for the general systems case.

The remainder of the paper is devoted to input/output stability and also input/output causality, on which the former concept rests. Essentially, a system (or rather, an input/output structure on a given behavior) is defined as causal if for any input with zero past, there exists a corresponding output with zero past. Strictly speaking, this definition only makes sense if the "past" contains an initial condition set for the zero-input behavior; we therefore restrict our attention to systems for which this behavior is time-autonomous. Bringing in some important results from the literature on PDEs, we demonstrate in section 4 that causality is a consequence of hyperbolicity of the $P$ operator. More strongly, we show that causality is equivalent to a property of the transfer matrix of the system, which is in turn equivalent to hyperbolicity of the $P^{c}$ operator in the description of the controllable part.

We then move on to stability, which is defined in terms of trajectories in $\mathcal{S}$ or in $\mathcal{S}^{\prime}$ having support in the half-space $t \geq 0$. An input/output structure is defined as stable if any causal output response to an input of this type is also necessarily of this type. The growth restrictions on these trajectories in the spatial directions convey the notion that they are "physically reasonable," and the growth restrictions in the temporal direction suggest stability. In section 5, we give some background results from the PDE literature on convolution operators for these special cases; these results effectively characterize input/output stability in this sense for the special case $p(\delta) y=u$. The general case requires some "structure theory" for behaviors over $\mathcal{S}_{+}$ (signals of $\mathcal{S}$ with support in the half-plane) and its dual space, which we develop in section 6. For completeness, using the same methodology we also develop analogous results for the spaces $\mathcal{S}$ and $\mathcal{S}^{\prime}$. In particular, for $n \leq 2$ and for the case where the $P$ operator is a single polynomial (covering the single-input single-output case in particular), we characterize inclusion of behaviors over $\mathcal{S}^{\prime}$ and freeness of variables over $\mathcal{S}$ (these being essentially dual problems). The more general case for $n>2$ is not proved, since the arguments used depend critically on proving a geometric property "ideal-convexity" of a particular set (the "bad frequency" set) in $\mathbb{C}^{n}$. To date, we have not been able to prove this property for $n \geq 2$; this is discussed in section 5.3.

Section 7 finally defines input/output stability, and characterizes it, again for the cases $n \leq 2$ or $P$ equal to a single polynomial (e.g., single-input single-output). It is shown that an input/output structure is both causal and stable (in terms of trajectories in $\mathcal{S}$ with zero past) if and only if the least common denominator of the transfer matrix satisfies the CV condition introduced in section 3, i.e., if and only if the system has no controllable unstable poles. Thus it appears that input/output stability may be equivalent to stability of the zero input behavior, as happens in the standard one-dimensional (1D) case. We also give a similar sufficient condition for stability with respect to trajectories in $\mathcal{S}^{\prime}$ with zero past; this requires no prior assumptions on the system. Extension of all results to the general $\mathrm{nD}$ case requires only a proof that the set of unstable frequencies is ideal-convex. We summarize in section 8 . 
2. Behaviors, classical spaces, and pole structure. We begin by briefly reviewing some concepts and results from the theory of $\mathrm{nD}$ behaviors; see, e.g., [22, 34] for the $1 \mathrm{D}$ case and, e.g., [17, 20, 35, 40] for general background on the continuous $\mathrm{nD}$ case.

2.1. Classical and associated spaces. We consider solutions to behaviors in the classical spaces from the theory of distributions and so begin by recalling these and associated spaces. We denote the classical spaces by $\mathcal{C}^{\infty}$ (smooth functions), $\mathcal{D}^{\prime}$ (distributions), $\mathcal{C}_{0}^{\infty}$ (compactly smooth functions), $\mathcal{E}^{\prime}$ (compactly supported distributions), $\mathcal{S}$ (rapidly decreasing functions), and $\mathcal{S}^{\prime}$ (tempered distributions). Here all functions and functionals are taken to be complex-valued. Recall (e.g., [23]) that rapidly decreasing functions are those functions which decay faster than any polynomial grows; a precise definition is given in section 5.1. The tempered distributions may be thought of as distributions that grow no faster than some polynomial (see, e.g., [8, sec. 7.1] for a detailed treatment of $\mathcal{S}$ and $\mathcal{S}^{\prime}$ ). Following [4] we also define, for any of the classical spaces $\mathcal{W}$, the spaces [4],

$$
\begin{aligned}
& \mathcal{W}_{+}:=\left\{w \in \mathcal{W} \mid \operatorname{supp} w \subseteq \mathbb{R}_{+}^{n}\right\} \\
& \mathcal{W}_{-}:=\left\{w \in \mathcal{W} \mid \operatorname{supp} w \subseteq \mathbb{R}_{-}^{n}\right\} \\
& \mathcal{W}_{\oplus}:=\mathcal{W} / \mathcal{W}_{-} \\
& \mathcal{W}_{\ominus}:=\mathcal{W} / \mathcal{W}_{+} .
\end{aligned}
$$

Here $\mathbb{R}_{+}:=\mathbb{R}^{n-1} \times[0,+\infty)$ and $\mathbb{R}_{-}:=\mathbb{R}^{n-1} \times(-\infty, 0]$.

The spaces $\mathcal{S}_{+}$and $\mathcal{S}_{\oplus}^{\prime}$ will prove particularly important in what follows. It is clear that any element $f \in \mathcal{S}_{\oplus}$ may be regarded as an element of the dual of $\mathcal{S}_{+}$, according to

$$
\langle f, \phi\rangle:=\langle\bar{f}, \iota(\phi)\rangle, \phi \in \mathcal{S}_{+},
$$

where $\bar{f}$ is any element of $\mathcal{S}^{\prime}$ which projects to $f$, and $\iota: \mathcal{S}_{+} \rightarrow \mathcal{S}$ is the natural inclusion. As noted in [4], $\mathcal{S}_{\oplus}^{\prime}$ is in fact equal to the dual space of $\mathcal{S}_{+}$.

Denote by $\mathbb{C}[s]$ the polynomial ring in $n$ indeterminates $s=s_{1}, \ldots, s_{n}$ with complex coefficients. We associate with any polynomial matrix $R=R(s) \in \mathbb{C}^{g \times q}$ the differential operator $R(\delta):=R\left(\partial / \partial x_{1}, \ldots, \partial / \partial x_{n}\right), x_{1}, \ldots, x_{n}$ being independent variables in the space $\mathbb{R}^{n}$. This operator maps $\mathcal{W}^{q}$ to $\mathcal{W}^{g}$ for any of the spaces $\mathcal{W}$ listed above (the action on factors $\mathcal{W}_{\oplus}, \mathcal{W}_{\ominus}$ being induced in the obvious way). We remark that in the theory of PDEs, it is more usual to consider operators in the form $w \mapsto R(1 / \imath) \partial) w$. For this reason, certain results in the theory of PDEs concerning the algebraic structure of operators change form in a straightforward way when translated into the current framework.

Recall also that for any of the classical pairs of dual spaces $\mathcal{W}, \mathcal{W}^{\prime}$, and any polynomial matrix $R \in \mathbb{C}[s]^{g \times q}$, the adjoint matrix $R^{*}(s):=R^{T}(-s)$ has the property that

$$
\langle R(\partial) f, \phi\rangle=\left\langle f, R^{*}(\partial) \phi\right\rangle
$$

for any $f \in \mathcal{W}^{q}, \phi \in\left(\mathcal{W}^{\prime}\right)^{g}$.

2.2. Behaviors, associated varieties, and time-autonomy. For any of the spaces $\mathcal{W}$ discussed in section 2.1 , and for a polynomial matrix $R \in \mathbb{C}[s]^{g \times q}$, 
denote as usual

$$
\begin{aligned}
\operatorname{ker}_{\mathcal{W}} R & =\left\{w \in \mathcal{W}^{q} \mid R(\delta) w=0\right\} \\
\operatorname{im}_{\mathcal{W}} R & =\left\{w \in \mathcal{W}^{g} \mid \exists l \in \mathcal{W}^{q} \text { s.t. } w=R(\partial) l\right\}
\end{aligned}
$$

In this situation, we say that $R$ is a kernel representation matrix of the behavior $\mathcal{B}=\operatorname{ker}_{\mathcal{R}} R . \mathcal{W}$ is referred to as the the signal space; the signal space of a behavior is taken to be $\mathcal{D}^{\prime}$ unless otherwise specified.

For the operator $R(\partial)$ or behavior $\operatorname{ker}_{\mathcal{D}^{\prime}} R$, the associated system module or module of formal quantities is defined as $\mathcal{M}:=\mathbb{C}[s]^{1 \times q} / \mathbb{C}[s]^{1 \times g} R$. This object is also standard in PDE theory, and some relationships between $\mathcal{M}$ and $\mathcal{B}$ are drawn out in [35]. In particular, the behavior $\mathcal{B}$ (for any signal space $\mathcal{W}$ ) may be identified with $\operatorname{Hom}_{\mathbb{C}[s]}(\mathcal{M}, \mathcal{W})[13,17]$.

Given a polynomial matrix $R \in \mathbb{C}[s]^{g \times q}$, recall that a universal or minimal left annihilator is a matrix $L \in \mathbb{C}[s]^{h \times g}$ for some $h$, such that the rows of $L$ generate the set of polynomial vectors $v$ with $v R=0$. Then the "fundamental principle" of EhrenpreisPalamodov states that $\operatorname{im}_{\mathcal{W}} R=\operatorname{ker} L$ for $\mathcal{W}=\mathcal{D}^{\prime}$ or $\mathcal{W}=\mathcal{C}^{\infty}$. Equivalently, these two signal spaces (modules) are injective. This property is a major component of a very rich relationship between system modules $\mathcal{M}$ and behaviors $\mathcal{B}$, introduced into behavioral theory by Oberst [17]. We will also use standard facts and results concerning the associated primes of $\mathcal{M}$; see, e.g., [3] for the background here.

Let $\mathcal{B}=\operatorname{ker}_{\mathcal{D}^{\prime}} R$ with $R \in \mathbb{C}[s]^{g \times q}$; denote by $\mathcal{V}(\mathcal{B})$ the characteristic variety

$$
\mathcal{V}(\mathcal{B}):=\left\{\zeta \in \mathbb{C}^{n} \mid \operatorname{rank} R(\zeta)<q\right\}
$$

which is well known to depend only on $\mathcal{B}$ and to be equal to the variety of the ideal

$$
\text { ann } \mathcal{M}:=\{r \in \mathbb{C}[s] \mid r x=0 \text { for all } x \in \mathcal{M}\} .
$$

The points of $\mathcal{V}(\mathcal{B})$ are precisely the frequencies $\zeta$ for which $\mathcal{B}$ admits polynomial exponential trajectories $p(x) \exp (\langle\zeta, x\rangle), p$ a polynomial function; see, e.g., $[18,37]$ for a discussion in the behavioral context. Since the associated primes of $\mathcal{M}$ include the primes minimal with respect to the property of including ann $\mathcal{M}, \mathcal{V}(\mathcal{B})$ is equal to the union of the varieties of the associated primes of $\mathcal{M}$.

We may further consider the projective closure $\overline{\mathcal{V}}$ of a variety $\mathcal{V}=\mathcal{V}(I)$ for some ideal $I$, which is the smallest projective variety containing $\mathcal{V}$ according to the inclusion $\mathbb{C}^{n} \rightarrow \mathbb{P C}^{n}, \zeta \mapsto(\zeta, 1) . \overline{\mathcal{V}}$ is the set of zeros of the homogeneous ideal equal to the set of all homogeneous polynomials in $\mathbb{C}[s, z]$ mapping $I$ under $p(s, z) \mapsto p(s, 1)$. The variety at infinity $\tilde{\mathcal{V}}$ is defined as the intersection of $\tilde{\mathcal{V}}$ with the "hyperplane at infinity" $\mathbb{C}^{n} \times 0 \subseteq \mathbb{P}^{n}$ (but $\tilde{\mathcal{V}}$ is regarded as an affine variety in $\mathbb{C}^{n}$ ). Since the hyperplane at infinity is defined by the additional equation $z=0, \tilde{\mathcal{V}}$ may easily be seen to equal the set of zeros of the principal parts $\operatorname{pr}(p)$ of all $p \in I$, where $\operatorname{pr}(p)$ is the sum of all terms of $p$ with the highest total degree. The variety at infinity may be computed, e.g., using Gröbner bases [3, sec. 15.10.5].

A vector $v \in \mathbb{R}^{n} \backslash 0$ is said to be a characteristic direction for $\mathcal{M}$ or for ann $\mathcal{M}$, or for the associated system of PDEs, if $v \in \tilde{\mathcal{V}}(\operatorname{ann} \mathcal{M})$; otherwise it is said to be noncharacteristic. Recall now the definition of time-autonomy due to Sasane, Thomas, and Willems [25].

Definition 2.1. A behavior $\mathcal{B}$ is called time-autonomous if any trajectory is determined by its restriction to the half-space $\left\{x \in \mathbb{R}^{n} \mid x_{n}<0\right.$. $\}$ The behavior is autonomous if the characteristic variety is not all of $\mathbb{C}^{n}$. 
Thus for a behavior $\mathcal{B}$ with signal space $\mathcal{D}^{\prime}$, time-autonomy is equivalent to the absence of nonzero solutions in $\mathcal{D}_{+}^{\prime}$, so means that if a trajectory is zero in the "past" $\left(\mathbb{R}_{-}^{n}\right)$ it must remain zero in the "future" $\left(\mathbb{R}_{+}^{n}\right)$.

Nonzero solutions over $\mathcal{D}_{+}^{\prime}$ or $\mathcal{C}_{+}^{\infty}$ (or more generally in a specified half-space) are null solutions. The following result characterizing their existence was first proved by Hormander [6] in the smooth, single polynomial case, and can be found in [15] for the smooth systems case and [16] for the distributional and smooth systems cases.

TheOrem 2.2. A behavior $\mathcal{B}$ in $\left(\mathcal{D}^{\prime}\right)^{q}$ or in $\left(\mathcal{C}^{\infty}\right)^{q}$ has no null solutions, i.e., is time-autonomous, if and only if $(0, \ldots, 0,1)$ is a noncharacteristic direction for the system.

Note. Recent work [27] has also given a characterization of time-autonomy for a class of $2 \mathrm{D}$ systems. Further consideration of how this relates to the above result is left as a topic for future research.

The (Willems) closure of a submodule $\mathcal{N}$ of $\mathbb{C}[s]^{1 \times q}$ with respect to a signal space $\mathcal{W}$ is defined $[20]$ as

$$
\mathcal{N}^{\perp \perp}:=\left\{v^{\prime} \in \mathbb{C}[s]^{1 \times q} \mid v^{\prime}(\partial) w=0 \text { for all } w \in \mathcal{N}^{\perp}\right\},
$$

where

$$
\mathcal{N}^{\perp}:=\left\{w \in \mathcal{W}^{q} \mid v(\partial) w=0 \text { for all } v \in \mathcal{N}\right\} .
$$

Notice that $\mathcal{N} \subseteq \mathcal{N}^{\perp \perp}$ and if $\mathcal{N}_{1}^{\perp} \subseteq \mathcal{N}_{2}^{\perp}$ are two behaviors, then $\mathcal{N}_{2}^{\perp \perp} \subseteq \mathcal{N}_{1}^{\perp \perp}$.

2.3. Pole structure. We now recall some results from [37] concerning the pole structure of $\mathrm{nD}$ behaviors. The material on input/output structures etc. may also be found in many other places in the literature.

Recall first that a (free) input/output structure $(x, y)$ on a behavior $\mathcal{B}$ with a general signal space $\mathcal{W}$ is a partition of the system variables into $m$ input variables $u$ and $p$ output variables $y$ with the properties that

1. the projection of the behavior onto the $u$ variables equals $\mathcal{W}^{m}$ (we say the variables $u$ are free over $\mathcal{W}$ ), and

2. the zero-input behavior

$$
\mathcal{B}_{0, y}:=\{(u, y) \in \mathcal{B} \mid u=0\}
$$

is autonomous, i.e., has no free variables.

For a given kernel representation, by writing the system equations in the form

$$
P(\partial) y=Q(\partial) u,
$$

we equivalently have that $P$ has full column rank and the rank of $(-Q, P)$ is equal to the rank of $Q$. When these conditions apply, there is a unique rational function matrix $G$ with $P G=Q$, called the transfer matrix.

The controllable part $\mathcal{B}^{c}$ of $\mathcal{B}$ defined as the (unique) maximal controllable subbehavior of $\mathcal{B}$, possesses the same input/output structures as $\mathcal{B}$ and admits the same transfer matrix with respect to any such input/output structure. We do not define controllability here but refer the reader to [20]. The zero-input behavior $\left(\mathcal{B}^{c}\right)_{0, y}$ of the controllable part has a special structure.

Lemma 2.3 (see [37, Thm. 5.3]). Let $\mathcal{B}^{c}$ be a controllable behavior with given input/output structure $(u, y)$. Let $\mathcal{M}^{\prime}$ be the system module associated to the zeroinput behavior $\left(\mathcal{B}^{c}\right)_{0, y}$. Then the associated primes of $\mathcal{M}^{\prime}$ are all principal, and the ideal ann $\mathcal{M}^{\prime}$ is generated by the least common denominator of the transfer matrix. 
For convenience, we will call a finitely generated module with the property that its associated primes are all principal a principal module; there is no standard term as far as we know.

The factor space $\mathcal{B} / \mathcal{B}^{c}$ has the structure of an "abstract behavior" as defined in [21]; it may be realized as any behavior of the form $R^{c}(\partial)(\mathcal{B})$, where $R^{c}$ is a kernel representation matrix of $\mathcal{B}^{c}$. The behavior $\mathcal{B} / \mathcal{B}^{c}$ is autonomous and is the natural analogue of the autonomous part in $1 \mathrm{D}$ behavioral theory. It may be identified with the set of all classes of mutually concatenable trajectories in $\mathcal{B}$ [40], and so this behavior may be called the "obstruction to controllability."

The pole variety, controllable pole variety, and uncontrollable pole variety of $\mathcal{B}$ (with a specified input/output structure) are defined, respectively, as $\mathcal{V}\left(\mathcal{B}_{0, y}\right)$, $\mathcal{V}\left(\left(\mathcal{B}^{c}\right)_{0, y}\right)$, and $\mathcal{V}\left(\mathcal{B} / \mathcal{B}^{c}\right)$. The points of the uncontrollable variety have an interpretation as input decoupling zeros, as discussed in [39]. The three sets are related as follows.

LEMMA 2.4. We have

$$
\begin{aligned}
& \mathcal{V}\left(\mathcal{B}_{0, y}\right)=\mathcal{V}\left(\left(\mathcal{B}^{c}\right)_{0, y}\right) \cup \mathcal{V}\left(\mathcal{B} / \mathcal{B}^{c}\right), \\
& \widetilde{\mathcal{V}}\left(\mathcal{B}_{0, y}\right)=\widetilde{\mathcal{V}}\left(\left(\mathcal{B}^{c}\right)_{0, y}\right) \cup \widetilde{\mathcal{V}}\left(\mathcal{B} / \mathcal{B}^{c}\right) .
\end{aligned}
$$

Proof. Equation (15) is derived in [37] from a standard general result. Equation (16) must also be a consequence of a standard result, but one that we have not found, so we derive it here. Let $\mathcal{M}, \mathcal{M}^{\prime}$, and $\mathcal{M}^{\prime \prime}$ denote the system modules corresponding, respectively, to the behaviors $\mathcal{B}_{0, y}, \mathcal{B} / \mathcal{B}^{c}$, and $\left(\mathcal{B}^{c}\right)_{0, y}$; then $\mathcal{M}^{\prime} \subseteq \mathcal{M}$ with factor $\mathcal{M}^{\prime \prime}$, and it is straightforward from this to see that

$$
\text { ann } \mathcal{M} \subseteq \text { ann } \mathcal{M}^{\prime} \cap \mathcal{M}^{\prime \prime} \subseteq \operatorname{rad} \text { ann } \mathcal{M},
$$

where rad denotes the radical of an ideal. Write

$$
J:=\{p r(d) \mid d \in \operatorname{ann} \mathcal{M}\}
$$

and define $J^{\prime}$ and $J^{\prime \prime}$ analogously with respect to $\mathcal{M}^{\prime}$ and $\mathcal{M}^{\prime \prime}$, respectively. Clearly, $J \subseteq J^{\prime} \cap J^{\prime \prime}$. Moreover, if $r \in J^{\prime} \cap J^{\prime \prime}$, say, $r=p r d_{1}=p r d_{2}$ with $d_{1} \in$ ann $\mathcal{M}^{\prime}, d_{2} \in$ ann $\mathcal{M}^{\prime \prime}$, then we find that $d_{1} d_{2} \in$ ann $\mathcal{M}$. We also see that for any two polynomials $p, q \in \mathbb{C}[s], \operatorname{pr}(p) \cdot \operatorname{pr}(q)=\operatorname{pr}(p q)$, and so $r^{2}=\operatorname{pr}\left(d_{1} d_{2}\right) \in J$, and so $r \in \operatorname{rad} J$. This proves that

$$
J \subseteq J^{\prime} \cap J^{\prime \prime} \subseteq \operatorname{rad} J
$$

Consequently, $\mathcal{V}(J)=\mathcal{V}\left(J^{\prime}\right) \cup \mathcal{V}\left(J^{\prime \prime}\right)$. However, $\mathcal{V}(J)=\tilde{\mathcal{J}}\left(\mathcal{B}_{0, y}\right)$, etc., so we have proved (16).

3. Stability of autonomous behaviors. In this section we consider an autonomous behavior $\mathcal{B}$ given by a kernel representation matrix $R$, which necessarily has full column rank $q$. Furthermore, we assume that one of the independent variables "time" $(t)$ is distinguished; without loss of generality we will always take this to be the last variable listed in the coordinate system for $\mathbb{R}^{n}$. Under what conditions should $\mathcal{B}$ be referred to as a "stable" behavior?

Stability in this context should mean that $\mathcal{B}$ contains no physically reasonable trajectories which grow in time at an unacceptably fast rate in some sense (e.g., which are unbounded). We might call this "stability with respect to the initial conditions." 
In $[20,30], \mathcal{B}$ was defined to be stable with respect to a cone $C \subseteq \mathbb{R}^{n}$ if every smooth trajectory of $\mathcal{B}$ tends to 0 along every half-line in $C$. This is characterized in $[20,30]$, subject to some minor assumptions, by the condition that no projection onto the real space of $\mathcal{V}(\mathcal{B})$ should lie in the polar cone of $C$ with positive distance from the boundary of this polar.

Let us consider the heat or diffusion equation in one spatial variable

$$
\frac{\partial^{2} w}{\partial x^{2}}=\frac{\partial w}{\partial t} .
$$

This system was used recently by Sasane in $[26,24]$ to motivate an alternative signal space to $\mathcal{C}^{\infty}, \mathcal{D}^{\prime}$; here we will consider it in a similar spirt. We find that the characteristic variety of the system (17) or of its behavior $\mathcal{B}$, is

$$
\mathcal{V}(\mathcal{B})=\left\{(\eta, \xi) \in \mathbb{C}^{2} \mid \eta^{2}=\xi\right\}
$$

and the behavior contains trajectories of the form

$$
\exp \left(\Re(\eta) x+\Re\left(\eta^{2}\right) t\right) \exp \left(\iota \Im(\eta) x+\iota \Im\left(\eta^{2}\right) t\right)
$$

for all $\eta \in \mathbb{C}$. Hence $\mathcal{B}$ contains trajectories which are unbounded on the positive $t$-axis, corresponding to the choices $\Re\left(\eta^{2}\right)>0$, and so is unstable in the sense introduced in $[20,30]$. However, note that if $\Re\left(\eta^{2}\right)>0$, then $\Re(\eta) \neq 0$; i.e., any solution which is unbounded on the $+t$-axis is also unbounded (indeed, exponentially growing) on the $x$-axis. In other words, the only way to get unbounded temporal behavior in this system is to start with exponentially growing initial spatial data! Indeed, we would prefer to consider the heat equation as "stable"; with no external input of heat, heat should diffuse in time and never blow up. In this paper, we take the view that the initial data and trajectories which are exponentially growing spatially are physically unrealistic. With these considerations in mind we introduce the following concept.

Definition 3.1. An autonomous behavior $\mathcal{B}$, or its characteristic variety $\mathcal{V}=$ $\mathcal{V}(\mathcal{B})$, is said to satisfy the CV condition if

$$
\mathcal{V} \cap \mathcal{X}^{+}=\emptyset, \mid \mathcal{X}^{+}:=\imath \mathbb{R}^{n-1} \times \overline{\mathbb{C}^{+}}
$$

where $\overline{\mathbb{C}^{+}}$denotes the closed right-half plane. We say that $\mathcal{B}$ or $\mathcal{V}$ satisfies the weak $\mathrm{CV}$ (WCV) condition if the same holds but for the open right-half plane $\mathbb{C}^{+}$instead of $\overline{\mathbb{C}^{+}}$. We also say that a polynomial or ideal satisfies the CV condition or satisfies the WCV condition if the corresponding condition is satisfied by the variety of the polynomial/ideal.

For later use we also define

$$
\mathcal{X}^{-}:=\imath \mathbb{R}^{n-1} \times \overline{\mathbb{C}^{-}},
$$

where $\overline{\mathbb{C}^{-}}$denotes the closed left-half plane.

Recalling the description of the points of the characteristic variety in terms of polynomial exponential trajectories, we note that a behavior satisfies the CV condition if and only if it contains no polynomial exponential trajectories which are bounded at $t=0$ (corresponding to the spatial frequency components being imaginary) but which do not decay along the positive $t$-axis (corresponding to the temporal frequency components being in $\overline{\mathbb{C}^{+}}$). This observation applies equally well to both complex and real-valued trajectories. We therefore think of points of $\mathcal{X}^{+}$as unstable frequencies. 
Similarly, a behavior satisfies the WCV condition if and only if it contains no polynomial exponential trajectories which are bounded at $t=0$ but grow faster than a polynomial in the $+t$-direction. Note that the behavior defined by the heat equation certainly satisfies the WCV condition, as if $\eta$ is imaginary, then $\Re\left(\eta^{2}\right)<0$ gives rise to a trajectory which is exponentially decaying in time.

As a working definition, we consider an autonomous behavior to be stable when it satisfies the CV condition. This attempts to capture the idea that a behavior is unstable when it contains trajectories which are well behaved at $t=0$ but do not decay to 0 as $t \rightarrow+\infty$. The concept of "stability with respect to initial conditions" also lies behind the definition of stability for 2D discrete systems given by Valcher [33] (there is little parallel in the mathematics of the continuous and discrete cases, but the underlying philosophy is the same). As we have seen, in terms of polynomial exponential trajectories Definition 3.1 seems very appropriate, and by the same considerations the CV condition is certainly necessary for stability in the general sense we have been indicating. Moreover, since the polynomial exponential solutions of a system are dense in the smooth solutions, at least in the case of complex coefficients [7, Thm. 7.6.14], it is not unreasonable to conjecture that the CV condition is also sufficient for this type of stability. However, this important problem remains open, and we rely on later sections to fully motivate the $\mathrm{CV}$ condition.

Let us cover the other basic classical examples for general $n$. We have seen that the behavior of the heat equation satisfies the WCV condition, though it does not satisfy the CV condition; this holds in any number of spatial dimensions. This behavior of the wave equation and of the gradient operator also satisfy the WCV condition but not the CV condition. The kernel of the Laplace operator does not satisfy either.

3.1. Hyperbolic systems. It is also interesting to note that the WCV condition implies the Gärding condition, which is necessary for hyperbolicity of an autonomous system given by a single polynomial. Here is the condition,

$$
\left\{\Re(\xi) \mid \exists \eta \in \imath \mathbb{R}^{n-1}, \xi, \eta \in \mathcal{V}\right\} \subseteq \mathbb{R} \text { is bounded above. }
$$

We now discuss hyperbolicity, giving the definition for the systems case which is more complex than the better known definition for a single polynomial. The following definition is identical to one of the equivalent definitions given by Nacinovich [16], adjusted only in respect of the fact that our systems are defined via $P(\partial) w=0$, whereas he uses the more standard $P((1 / \imath) \partial) w=0$. Also we have specialized the definition to hyperbolicity in a fixed direction.

Definition 3.2. A system $P(\partial) w=0$, operator $P(\partial)$, associated system module $\mathcal{M}$, or behavior $\mathcal{B}$, is called hyperbolic (in the direction $t$ ) if for every associated prime $I$ of $\mathcal{M}$ we can find a constant $0<c<1$ such that (where $\Re(\eta, \xi)$ denotes the real part vector of the complex $n+1$ tuple $(\eta, \xi))$

$$
\Re(\xi) \leq c|\Re(\eta, \xi)|+c^{-1} \text { for every }(\eta, \xi) \in \mathcal{V}(I) .
$$

The following result, also adapted from [16], links the definition to the more familiar one for a single polynomial.

Theorem 3.3. Let $P \in \mathbb{C}[s]^{g \times q}$, and let $\mathcal{M}$ be the system module, i.e., $\mathcal{M}=$ $\mathbb{C}[s]^{1 \times q} / \mathbb{C}[s]^{1 \times g} P$. Suppose that $\mathcal{M}$ is principal. Then $P(\partial)$ is hyperbolic if and only if $(0, \ldots, 0,1)$ is a noncharacteristic direction for the system $P(\partial) y=0$, and the Gårding condition (20) holds for the characteristic variety of the system.

Proof. We refer to the remark following [16, Prop. 6.1], which states that when the associated primes of $\mathcal{M}$ are all principal, hyperbolicity is equivalent to the requirement 
that $(0, \ldots, 0,1)$ be noncharacteristic, together with the Gårding condition for each variety $\mathcal{V}(I), I$ an associated prime of $\mathcal{M}$. Since the number of associated primes is finite, the latter condition is, however, equivalent to the Gårding condition for the characteristic variety itself.

It is easy to see that the conditions of Theorem 3.3 hold in particular when $P$ is a single polynomial, as expected. Note from Lemma 2.3 that this condition is also met for the module corresponding to the zero-input behavior of any controllable behavior.

We remark that hyperbolicity is equivalent to solvability of the "noncharacteristic" Cauchy problem in many different formulations [9, 10], which is of great importance and deserves investigation in the context of control systems theory. Essentially, hyperbolicity allows the unique continuation of initial data in a large class on $t=0$ to trajectories on the half-space $t \geq 0$. We will note in the next section its connections to causality.

Note also that hyperbolic behaviors are in particular time-autonomous (in the general case this is a consequence of Theorem 4.2 in the next section). Next, we link the WCV condition to hyperbolicity.

LEMma 3.4. Let $P(\partial)$ be a partial differential operator with kernel $\mathcal{B}$ and system module $\mathcal{M}$. Suppose that $\mathcal{M}$ is principal and that $\mathcal{B}$ satisfies the $W C V$ condition and is time-autonomous. Then the system is hyperbolic and therefore admits a solution to the noncharacteristic Cauchy problem.

In the case where $\mathcal{M}$ is principal, the $\mathrm{CV}$ property, together with time-autonomy, is of course a much stronger property than hyperbolicity; for example, in two dimensions the kernel of the operator $(\partial / \partial t-1)$ is hyperbolic but does not satisfy the WCV condition. The relationship between these two properties will become clearer when we examine stable input/output structures. This, however, will require us to consider causality in the continuous space-time input/output framework.

4. Causal input/output structures. We are interested in this section with the question of when a given input/output structure is causal. Following Zampieri [38] for the discrete case (in which the past and future are defined with respect to a cone), we introduce the following definition of causality.

DEFINITION 4.1. Suppose that $(u, y)$ is an input/output structure on $\mathcal{B}$ and $\mathcal{B}_{0, y}$ is time-autonomous. Then the input/output structure is said to be causal (with respect to $\mathcal{C}^{\infty}$ ) if for any smooth input $u$ with support in $\mathbb{R}_{+}^{n}$, there exists a smooth output $y$ (necessarily unique) with support in $\mathbb{R}_{+}^{n}$, such that $(u, y) \in \mathcal{B}$.

Causality with respect to $\mathcal{D}^{\prime}$ may also be defined in the obvious way; where no solution type is specified, we take causality to be meant in the smooth sense.

Thus causality indicates that for any input $u$ with zero past, there is a corresponding output $y$ (intuitively, the output corresponding to zero initial conditions) with a past which is determined by that of $u$, and is therefore also zero. Notice that this interpretation of Definition 4.1 only makes complete sense if the complementary half-space $\mathbb{R}_{-}^{n}$ contains the domain of a complete set of Cauchy data for $\mathcal{B}_{0, y}$, and this is the reason for insisting a priori that $\mathcal{B}_{0, y}$ be time-autonomous. Time-autonomy of $\mathcal{B}_{0, y}$ clearly means that an output trajectory is entirely determined by its value in $\mathbb{R}_{-}^{n}$, together with the input trajectory. Physical systems should of course always be causal in the intuitive sense, but not all autonomous behaviors arising in the physical context are time-autonomous. For example, the behavior defined by the heat equation (18) is not time-autonomous, though in a more restricted and perhaps physically better motivated sense, it is (see [24]). A general solution of the heat equation is, however, not uniquely defined by its past! - essentially because an input may be supplied via 
a boundary condition. For time-autonomous $\mathcal{B}_{0, y}$, however, causality in the sense of Definition 4.1 captures the intuitive concept.

Hyperbolicity is intimately connected to causality. To demonstrate this, we give some results essentially taken from [16]; these results are very well known in the PDE community for the case of a single polynomial operator (see, e.g., [9, Thm. 12.5.4]), in which case the matrix $L$ below is 0 .

TheOREM 4.2. Let $P \in \mathbb{C}[s]^{g \times q}$ with universal left annihilator matrix $L \in$ $\mathbb{C}[s]^{h \times g}$. The following are equivalent:

1. $P(\partial)$ is hyperbolic.

2. The system

$$
P(\partial) y=u
$$

has a unique solution $y \in\left(\mathcal{C}_{+}^{\infty}\right)^{p}$ for all $u \in \operatorname{ker}_{\mathcal{C}_{+}^{\infty}} L$.

3. The system (21) has a unique solution $y \in\left(\mathcal{D}_{+}^{\prime}\right)^{p}$ for all $u \in \operatorname{ker}_{\mathcal{D}_{+}^{\prime}} L$.

Proof. In [16, Thm. 5.1], it is stated that $P(\partial)$ is hyperbolic if and only if $\operatorname{Ext}_{\mathbb{C}[s]}^{i}\left(\mathcal{M}, \mathcal{C}_{+}^{\infty}\right)=0, i=0,1$. For $i=0$ this means that $P(\partial)$ admits no smooth null solutions; for $i=1$ it means that the sequence

$$
\left(\mathcal{C}_{+}^{\prime}\right)^{p} \stackrel{P(\partial)}{\rightarrow}\left(\mathcal{C}_{+}^{\infty}\right)^{g} \stackrel{L(\partial)}{\rightarrow}\left(\mathcal{C}_{+}^{\infty}\right)^{h}
$$

is exact, which together with condition 2 [16, Thm. 5.2] gives the result for distributions, which establishes the equivalence of $1-3$.

Notice that Theorem 4.2 in particular gives a restricted form of the "fundamental principle" - over $\mathcal{C}_{+}^{\infty}$ or $\mathcal{D}_{+}^{\prime}$ when $P$ (or its module) is hyperbolic, the system (21) has a solution $y$ for given $u$ if and only if $u$ satisfies the necessary "compatibility conditions." Indeed, the results of Nacinovich characterize hyperbolicity in terms of the vanishing of $\operatorname{Ext}_{\mathbb{C}[s]}^{i}$, as indicated in the proof above.

Moreover, at least in the case of a single polynomial [9, Thm. 12.5.4], hyperbolicity guarantees the existence of a fundamental solution to the system (21) having support in $\mathbb{R}_{+}^{n}$, and in both smooth and distributional cases the causal input-to-output map is given simply by convolution with the fundamental solution, which in the current context we can also refer to as the impulse response. As discussed in [30], hyperbolic systems are in this sense, therefore, the natural analogue of standard 1D (lumped) systems.

In [30], Shankar considers causality in a slightly different sense, namely to mean the existence of a continuous linear shift-invariant map from the set of smooth inputs with support in some cone contained in $\mathbb{R}_{+}^{n}$, to the set of outputs of the same type. For the system (21) with $P$ equal to a single polynomial $p$, he proves that the existence of such a causal input-to-output map guarantees time-autonomy of the zero-input behavior and thereby (using Theorem 4.2) hyperbolicity of $p$ also.

It is easy to see that hyperbolicity is still sufficient for results of this type when a $Q$ term is added to the equations.

Corollary 4.3. Let $\mathcal{B}$ be a behavior defined by the equations

$$
P(\partial) y=Q(\partial) u
$$

forming an input/output structure. If $P$ is a hyperbolic operator, then $\mathcal{B}_{0, y}$ is timeautonomous and the input/output structure is causal.

Proof. Suppose that $P$ is hyperbolic. Time-autonomy of $\mathcal{B}_{0, y}$ is immediate from the uniqueness of solutions $y$ given in Theorem 4.2, and causality follows from this result also. 
It is open to discussion as to whether the converse of Corollary 4.3 holds. The next lemma shows that under a simple assumption, all the trajectories of $\mathcal{B}$ with support in $\mathcal{D}_{+}^{\prime}$ are contained in the controllable part. We will then use this reasoning to demonstrate that $\mathcal{B}$ and $\mathcal{B}^{c}$ have the same causal input/output structures.

LEMma 4.4. Let $\mathcal{B}$ be a behavior in $\left(\mathcal{D}^{\prime}\right)^{q}$. If $\mathcal{B} / \mathcal{B}^{c}$ is time-autonomous, then $\mathcal{B} \cap\left(\mathcal{D}^{\prime}\right)^{q}=\mathcal{B}^{c} \cap\left(\mathcal{D}_{+}^{\prime}\right)^{q}$.

Proof. Suppose that $\mathcal{B} / \mathcal{B}^{c}$ is time-autonomous. The inclusion $\supseteq$ is trivial. Now suppose $w \in \mathcal{B} \cap\left(\mathcal{D}_{+}^{\prime}\right)^{q}$, and let $R^{c}$ be a kernel representation matrix of $\mathcal{B}^{c}$. Since $\mathcal{B} / \mathcal{B}^{c}$ is time-autonomous, $R^{c}(\partial) w \in R^{c}(\partial)(\mathcal{B}) \cong \mathcal{B} / \mathcal{B}^{c}$ must vanish, so $w \in \mathcal{B}^{c}$.

An analogous argument may be used to generalize Zampieri's Lemma 3.3 and, hence, other results in [38] from the $2 \mathrm{D}$ to $\mathrm{nD}$ discrete case.

COROLlary 4.5. Let $\mathcal{B}$ be a behavior with controllable part $\mathcal{B}^{c}$ and a given input/output structure $(u, y)$ (which is necessarily an input/output structure on $\mathcal{B}^{c}$ also). Then the following hold:

1. $\mathcal{B}_{0, y}$ is time-autonomous if and only if both $\mathcal{B} / \mathcal{B}^{c}$ and $\left(\mathcal{B}^{c}\right)_{0, y}$ are.

2. Under the equivalent conditions of claim $1,(u, y)$ is a causal input/output structure on $\mathcal{B}$ if and only if it is a causal input/output structure on $\mathcal{B}^{c}$.

Proof.

1. This claim is immediate from Theorem 2.2 together with Lemma 2.4.

2. Suppose that the conditions of claim 1 hold. If $(u, y)$ is causal for $\mathcal{B}^{c}$, then it is trivial that it is also causal for $\mathcal{B}$, and the converse follows directly from Lemma 4.4 .

Corollary 4.3, Lemma 4.4, and Corollary 4.5 apply to causality in both the smooth and distributional senses.

One consequence of Corollary 4.5 is that when causality of an input/output structure is defined (i.e., when the zero-input behavior is time-autonomous), whether or not it holds is determined purely by the controllable part of the behavior and therefore by the transfer matrix. This motivates the following definition.

Definition 4.6. Call a transfer matrix $G$ causal if its least common denominator is hyperbolic, stable if this polynomial obeys the CV condition, and weakly stable if this polynomial obeys the WCV condition.

Notice that for $n=1$, stability of $G$ agrees with the classical concept, and causality of $G$ is automatic.

Suppose we are given a behavior $\mathcal{B}$ with input/output structure $(u, y)$ and transfer matrix $G$. Due to Lemma $2.3, G$ is causal if and only if $\left(\mathcal{B}^{c}\right)_{0, y}$ is hyperbolic. Similarly, $G$ is stable if and only if $\left(\mathcal{B}^{c}\right)_{0, y}$ obeys the CV condition. The following new result shows that causality of $G$ corresponds to causality of the corresponding input/output structures when the latter are defined.

THEOREM 4.7. Suppose that $\mathcal{B}$ is a behavior with an input/output structure such that $\mathcal{B}_{0, y}$ is time-autonomous. Then the input/output structure is causal with respect to $\mathcal{C}^{\infty}$ if and only if the associated transfer matrix is causal. These conditions imply that the input/output structure is causal with respect to $\mathcal{D}^{\prime}$.

Proof. Let $\mathcal{B}$ be given with $\mathcal{B}_{0, y}$ time-autonomous and let $P(\partial) y=Q^{c}(\partial) u$ be a description of the controllable part of $\mathcal{B}^{c}$. Write $\mathcal{M}$ for the system module of the behavior $\mathcal{B}^{c}$ and $\mathcal{M}^{\prime}$ for the system module of the operator $P^{c}(\partial)$, corresponding to the behavior $\left(\mathcal{B}^{c}\right)_{0, y}$. We have an exact sequence (e.g., [37])

$$
0 \rightarrow F \rightarrow \mathcal{M} \rightarrow \mathcal{M}^{\prime} \rightarrow 0
$$

for a free submodule $F$ of $\mathcal{M}$, with rank equal to the number of inputs $m$. From this 
we obtain the long exact sequence in Ext (see, e.g., [3]),

$$
\begin{gathered}
0 \rightarrow \operatorname{Hom}_{\mathbb{C}[s]}\left(\mathcal{M}^{\prime}, \mathcal{C}_{+}^{\infty}\right) \rightarrow \operatorname{Hom}_{\mathbb{C}[s]}\left(\mathcal{M}, \mathcal{C}_{+}^{\infty}\right) \stackrel{\rho}{\rightarrow} \operatorname{Hom}_{\mathbb{C}[s]}\left(F, \mathcal{C}_{+}^{\infty}\right) \\
\rightarrow \operatorname{Ext}_{\mathbb{C}[s]}^{1}\left(\mathcal{M}^{\prime}, \mathcal{C}_{+}^{\infty}\right) \rightarrow \operatorname{Ext}_{\mathbb{C}[s]}^{1}\left(\mathcal{M}, \mathcal{C}_{+}^{\infty}\right) \rightarrow 0
\end{gathered}
$$

the last " 0 " term occurring since $F$ is free. Recall that $\operatorname{Hom}_{\mathbb{C}[s]}\left(\mathcal{M}^{\prime}, \mathcal{C}_{+}^{\infty}\right)$ is identified with the $\mathcal{C}_{+}^{\infty}$ solutions in $\left(\mathcal{B}^{c}\right)_{0, y}$ and $\operatorname{Hom}_{\mathbb{C}[s]}\left(\mathcal{M}, \mathcal{C}_{+}^{\infty}\right)$ with the $\mathcal{C}_{+}^{\infty}$ solutions in $\mathcal{B}^{c}$. Also, $\operatorname{Hom}_{\mathbb{C}[s]}\left(F, \mathcal{C}_{+}^{\infty}\right)=\left(\mathcal{C}_{+}^{\infty}\right)^{m}$ and the map $\rho$ is the projection map $(u, y) \in$ $\left(\mathcal{C}_{+}^{\infty}\right)^{m+p} \mapsto u$. Since $\mathcal{B}^{c}$ is controllable, $\mathcal{M}$ is torsionfree [20]. Furthermore, we have an exact sequence

$$
0 \rightarrow \mathcal{C}_{+}^{\infty} \rightarrow \mathcal{C} \rightarrow \mathcal{C}_{\ominus}^{\infty} \rightarrow 0 .
$$

Note that $\mathcal{C}_{\ominus}^{\infty}$ is the set of restrictions of smooth functions to the set $\mathbb{R}_{-}^{n}$; it is therefore the direct limit as $\epsilon \mapsto 0$ of the sets $\mathcal{C}^{\infty}\left(H_{\epsilon}\right)$ on $H_{\epsilon}=\left\{x \in \mathbb{R}^{n}, x_{n}<\epsilon\right\}$ for $\epsilon>0$. Since $H_{\epsilon}$ is convex, $\mathcal{C}^{\infty}\left(H_{\epsilon}\right)$ is known to be an injective module (e.g., [19, Cor. VII.8.4]), and now $\mathcal{C}_{\ominus}^{\infty}$ is injective as the direct limits of injectives (e.g., [11, Thm. (3.46)]). Therefore $\mathcal{C}_{+}^{\infty}$ has injective dimension 1 , and so, $\mathcal{M}$ being torsionfree, $\operatorname{Ext}_{\mathbb{C}[s]}^{1}\left(\mathcal{M}, \mathcal{C}_{+}^{\infty}\right)=0$ by $[36$, Thm. 4.8]. From (22), we now see that the cokernel of $\rho$ equals $\operatorname{Ext}_{\mathbb{C}[s]}^{1}\left(\mathcal{M}^{\prime}, \mathcal{C}_{+}^{\infty}\right)$.

Suppose now that $(x, y)$ is causal for $\mathcal{B}$. Then by Corollary 4.5 , it is also causal for $\mathcal{B}^{c}$, so the map $\rho$ is surjective, due to which $\operatorname{Ext}_{\mathbb{C}[s]}^{1}\left(\mathcal{M}^{\prime}, \mathcal{C}_{+}^{\infty}\right)=0$. Also, as $\mathcal{B}_{0, y}$ is timeautonomous, $(\mathcal{B})_{0, y}^{c}$ is time-autonomous by Corollary 4.5, and so $\operatorname{Ext}_{\mathbb{C}[s]}^{0}\left(\mathcal{M}^{\prime}, \mathcal{C}_{+}^{\infty}\right)=0$ also. By Theorem 4.2, $P^{c}(\partial)$ is hyperbolic, which means that $G$ is causal.

Conversely, suppose that $G$ is causal. As observed preceding the theorem, $P^{c}(\partial)$ is hyperbolic and so $\operatorname{Ext}_{\mathbb{C}[s]}^{1}\left(\mathcal{M}^{\prime}, \mathcal{C}_{+}^{\infty}\right)$ vanishes by Theorem 4.2. Thus the map $\rho$ is surjective, so the variables $u$ are free over $\mathcal{C}_{+}^{\infty}$, i.e., $(u, y)$ is causal for $\mathcal{B}^{c}$ and so for $\mathcal{B}$ by Corollary 4.5. This converse argument also applies to distributional solutions.

In particular, Theorem 4.7 establishes that causality of a given input/output structure may be tested (when it is defined) merely by looking at the least common denominator $d$ of the transfer matrix $G$. In fact, since the prior condition of timeautonomy enforces that $\left(\mathcal{B}^{c}\right)_{0, y}$ be time-autonomous (by Corollary 4.5) and therefore that $(0, \ldots, 0,1)$ be noncharacteristic for $d$, we have that $(u, y)$ is causal if and only if $d$ satisfies the Garding condition. Unfortunately, it is not immediately clear how the condition may be tested. One possibly tractable necessary and sufficient condition is given in [16, Prop. 6.1]; $\mathcal{M}$ is hyperbolic if and only if $(0, \ldots, 0,-1)$ does not appear in any of the asymptotic cones (at infinity) of the real parts of the varieties of the associated primes of $\mathcal{M}$; for brevity we omit a precise description.

We remark also that a well-known necessary condition for hyperbolicity of $d$ is that the principal part of $\operatorname{pr}(d)$ of $d$ itself be hyperbolic (e.g., [9, Thm. 12.4.2]). The Gårding condition on $\operatorname{pr}(d)$ is equivalent to requiring that the roots $\zeta$ of $\operatorname{pr}(d)(\nu, \zeta)$ all be real for any real $\nu \neq 0$ (e.g., [9, Thm. 12.4.3]). Any further analysis of computational issues is outside the scope of this paper.

5. Convolution operators and ideal convexity. Before we tackle the subject of stable input/output structures, we need to explore two areas of background material. This first is convolution operators and Fourier transforms on the classical and other related spaces, as developed by Gindikin and Volevich [4]. This will lead us to a necessary and sufficient condition for input/output stability of the system 
$p(\partial) y=u$. The second area is "ideal-convexity" of a region in complex space, which is a necessary property for the extension of certain results from polynomials to ideals (and thereby general systems).

5.1. Convolution operators on $\mathcal{S}, \mathcal{S}^{\prime}$. The following material is largely taken from $\left[4\right.$, secs. 1.1-1.2]. For $s \in \mathbb{N}$ and $l \in \mathbb{R}$, let $\mathcal{C}_{(l)}^{(s)}$ denote the space of $s$-times continuously differentiable functions $f$ on $\mathbb{R}^{n}$ with finite Hölder norm

$$
|f|_{(l)}^{(s)}:=\sup _{x \in \mathbb{R}^{n}, \alpha \in \mathbb{N}^{n},|\alpha| \leq s}\left(1+x^{2}\right)^{l / 2}\left|\partial^{\alpha} f(x)\right| .
$$

Here $|\alpha|$ denotes the total of the components of $\alpha$, and $\delta^{\alpha}$ is a shorthand for the operator $p(\partial)$, where $p=\left(s_{1}^{\alpha_{1}} s_{2}^{\alpha_{2}}, \ldots, s_{n}^{\alpha_{n}}\right)$. The following elementary lemma is not in [4] but will prove useful.

Lemma 5.1. If $f, g \in \mathcal{C}_{l / 2}^{(s)}$ for some $s, l$, then $f g \in \mathcal{C}_{l}^{(s)}$.

Proof. Suppose that $f$ and $g$ are in $\mathcal{C}_{(l / 2)}^{(s)}$. We have

$\sup _{x \in \mathbb{R}^{n},|\alpha| \leq s}\left(1+x^{2}\right)^{l / 2}\left|\partial^{\alpha}(f g)(x)\right|=\sup _{x \in \mathbb{R}^{n},|\alpha| \leq s}\left(1+x^{2}\right)^{l / 2}\left|\sum_{i \leq \alpha} \beta_{i, \alpha}\left(\partial^{i} f\right)(x)\left(\partial^{\alpha-i} g\right)(x)\right|$,

where the sum on the right-hand side is taken over all multi-indices $i$ which are, componentwise, less than or equal to $\alpha$, and $\beta_{i, \alpha}$ are constants depending only on $i, \alpha$. Hence we obtain

$$
\begin{aligned}
\sup _{x \in \mathbb{R}^{n},|\alpha| \leq s}\left(1+x^{2}\right)^{l / 2}\left|\partial^{\alpha}(f g)(x)\right| \leq & \sup _{x \in \mathbb{R}^{n},|\alpha| \leq s} \sum_{i \leq \alpha}\left(1+x^{2}\right)^{l / 2}\left|\beta_{i, \alpha}\right|\left|\left(\partial^{i} f\right)(x)\right|\left|\left(\partial^{\alpha-i} g\right)(x)\right| \\
\leq & \max _{|\alpha| \leq s} \sum_{i \leq \alpha}\left|\beta_{i, \alpha}\right|\left(\sup _{x \in \mathbb{R}^{n}}\left(1+x^{2}\right)^{l / 4}\left|\left(\partial^{i} f\right)(x)\right|\right) \\
& \times\left(\sup _{x \in \mathbb{R}^{n}}\left(1+x^{2}\right)^{l / 4}\left|\left(\partial^{\alpha-i} g\right)(x)\right|\right),
\end{aligned}
$$

which is finite since for any $\alpha$ the sum given is a finite linear combination of terms which are finite by supposition. Therefore $f g \in \mathcal{C}_{(l)}^{(s)}$ as required.

Recall that $\mathcal{S}$ is defined as the intersection of all the spaces $\mathcal{C}_{(l)}^{(s)}$. Also of interest is the set

$$
\mathcal{L}:=\bigcap_{s} \bigcup_{l} \mathcal{C}_{(l)}^{(s)}
$$

which is the set of all smooth functions, each derivative of which grows no faster than some power of $x$ (which power may depend on the derivative), and

$$
\mathcal{O}:=\bigcup_{l} \bigcap_{s} \mathcal{C}_{(l)}^{(s)}
$$

the set of all smooth functions, each derivative of which grows no faster than some power of $x$ which is independent of the derivative. ( " $\mathcal{M}$ " is used rather than " $\mathcal{L}$ " in [4].) $\mathcal{O}$ may be thought of as the set of (at most) slowly growing smooth functions; its dual space is denoted by $\mathcal{O}^{\prime}$ and may be thought of as the space of rapidly decreasing distributions. Clearly $\mathcal{S} \subseteq \mathcal{O} \subseteq \mathcal{L} \subseteq \mathcal{C}^{\infty}$. We collect some basic facts and results from [4, sec. 1.1]. 
LEMMA 5.2.

1. $\mathcal{L}$ is a ring with respect to multiplication.

2. $\mathcal{S}$ is closed under multiplication by elements of $\mathcal{L}$.

3. $\mathcal{F}(\mathcal{S})=\mathcal{S}, \mathcal{F}\left(\mathcal{S}^{\prime}\right)=\mathcal{S}^{\prime}$, and $\mathcal{F}\left(\mathcal{O}^{\prime}\right)=\mathcal{L}$, where $\mathcal{F}$ denotes Fourier transform.

4. Let $p$ be a polynomial. The equation

$$
p(\partial) y=u
$$

is uniquely solvable for $y \in \mathcal{S}\left(\right.$ resp., $\left.\mathcal{O}, \mathcal{S}^{\prime}, \mathcal{O}^{\prime}\right)$ for any $u \in \mathcal{S}\left(\right.$ resp., $\left.\mathcal{O}, \mathcal{S}^{\prime}, \mathcal{O}^{\prime}\right)$, if and only if $p$ has no imaginary roots.

5. Any polynomial $p$ is in $\mathcal{L}$, and the function $p(\imath \zeta)$ of $\zeta$ has an inverse in $\mathcal{L}$ if and only if $p$ has no imaginary roots.

Proof. The last claim is not given explicitly in [4]; note that polynomials are contained in $\mathcal{O} \subseteq \mathcal{L}$. That the inverse of $p(\imath \zeta)$ of $\zeta$ is in $\mathcal{L}$ if and only if $p$ has no imaginary roots is implicit in the argument of [4, sec. 1.1.5]; we provide an argument based on their explicit results. By claim 4 , the polynomial $p$ has no imaginary roots if and only if the convolutional equation $p(\partial) y=u$ is uniquely solvable for $y \in \mathcal{O}^{\prime}$ for any $u \in \mathcal{O}^{\prime}$. By [4, Thm. 1.1.5], this holds if and only if there is a fundamental solution $E \in \mathcal{O}^{\prime}$ with $(p(\partial) \delta) * E=E *(p(\partial) \delta)=\delta$, where $\delta$ is the Dirac delta and $*$ denotes convolution. By Fourier transform (use claim 3), this is equivalent to invertibility of $p(\imath \zeta)$ in $\mathcal{L}$.

5.2. Convolution operators on $\mathcal{S}_{+}, \mathcal{S}_{+}^{\prime}$. Still following [4], we now introduce the spaces $\mathcal{C}_{(l)+}^{(s)}$ of all functions in $\mathcal{C}_{(l)}^{(s)}$ with support in $\mathbb{R}_{+}^{n}$ and define $\mathcal{S}_{+}, \mathcal{S}_{+}^{\prime}, \mathcal{S}_{\oplus}^{\prime}$, etc., as before. $\mathcal{O}_{+}$and $\mathcal{O}_{+}^{\prime}$ are defined analogously. We have that $\mathcal{S}_{\oplus}^{\prime}$ is the dual space of $\mathcal{S}_{+}$and $\mathcal{S}_{+}^{\prime}$ is dual to $\mathcal{S}_{\oplus}$.

Now for any Banach space $B$ of functions $(\nu, \sigma) \in \mathbb{R}^{(n-1)+1}$ with norm $\phi \mapsto|\phi|_{B}$, denote by $B^{+}$the space of functions $f$ of $(\nu, \xi) \in \mathbb{R}^{n-1} \times \mathbb{C}, \xi=\sigma+\imath \rho$, with the following properties:

1. For each $\rho \leq 0$, the functions $f_{\rho}=f(\cdot, \cdot+\imath \rho)$ are in $B$, and the map $(-\infty, 0] \mapsto$ $B, \rho \mapsto f_{\rho}$ is continuous.

2. For each $\nu \in \mathbb{R}^{n-1}$, the functions $f_{\nu}=f(\nu, \cdot)$ are functions holomorphic in $\mathbb{C}_{-}$.

3. The norm $\sup _{\rho<0}\left|f_{\rho}\right|_{B}$ is finite.

A space $B^{-}$may be defined analogously by changing the sign of $\rho$ in conditions 1 and 3 and changing $\mathbb{C}_{-}$for $\mathbb{C}_{+}$in condition 2. Note that if $f(s) \in B^{+}$, then $F(-s) \in B^{-}$and vice versa, provided that $B$ is preserved by the same operation.

Now we define

$$
\begin{aligned}
& \mathcal{S}^{+}:=\bigcap_{s, l} \mathcal{C}_{(l)}^{(s)+}, \\
& \mathcal{L}^{+}:=\bigcap_{s} \bigcup_{l} \mathcal{C}_{(l)}^{(s)+} .
\end{aligned}
$$

Spaces $\mathcal{S}^{-}$and $\mathcal{L}^{-}$may be defined analogously, and again if $f(s) \in \mathcal{L}^{+}$, then $f(-s) \in$ $\mathcal{L}^{-}$and vice versa.

The interest in these spaces comes from the following collection of points, from [4] except where proof is given below. 
LEMMA 5.3.

1. $\mathcal{L}^{+}$is closed under multiplication.

2. $\mathcal{S}^{+}$is closed under multiplication by elements of $\mathcal{L}^{+}$and this multiplication rule is associative.

3. $\mathcal{F}\left(\mathcal{S}_{+}\right)=\mathcal{S}^{+}$and $\mathcal{F}\left(\mathcal{O}_{+}^{\prime}\right)=\mathcal{L}^{+}$.

4. Let $p$ be a polynomial. The equation

$$
p(\partial) y=u
$$

is uniquely solvable for $y \in \mathcal{S}_{+}^{\prime}$ for any $u \in \mathcal{S}_{+}^{\prime}$ if and only if $p$ has no roots in $\mathcal{X}^{+}=\mathbb{R}^{n-1} \times \overline{\mathbb{C}_{+}}$.

5. The equation

$$
p(\partial) y=u
$$

is uniquely solvable for $y \in \mathcal{S}_{+}^{\prime}$ for any $u \in \mathcal{S}_{+}^{\prime}$ if and only if $p$ has no roots in $\mathbb{R}^{n-1} \times \overline{\mathbb{C}_{+}}$.

6. Any polynomial $p$ is in $\mathcal{L}^{+}$and the function $1 / p(\imath \zeta)$ of $\zeta$ is in $\mathcal{L}^{+}$if and only if $p$ has no roots in $\mathcal{X}^{+}$.

Proof.

1. Note that multiplication is defined since the elements of $\mathcal{L}^{+}$are functions. It follows from Lemma 5.1 that if $f, g \in \mathcal{C}_{(l / 2)}^{(s)+}$ for some $l \in \mathbb{R}$, then $f g \in \mathcal{C}_{(l / 2)}^{(s)+}$.

Hence if $f, g \in \mathcal{L}^{+}$, then for any $s$ there exists $l$ with $f, g \in \mathcal{C}_{(l)}^{(s)+}$, and so $f g \in \mathcal{C}_{(l)}^{(s)+}$. Therefore $f g \in \mathcal{L}^{+}$.

2. The first point is given in $[4$, sec. 1.2.2], and the second is immediate as $\mathcal{L}^{+}, \mathcal{S}^{+} \subseteq \mathcal{C}^{\infty}$.

3. These identities are (21) and (24) in [4, sec. 1].

4. This is Theorem 2(i) in [4, sec. 1.2.5].

5. This is Theorem 2(ii) in [4, sec. 1.2.5].

6. That polynomials are in $\mathcal{L}^{+}$follows from claim 3 , as polynomials are Fourier transforms of distributions with support at 0 , which are therefore in $\mathcal{O}_{+}^{\prime}$. Also from [4, Thm. 2(i), sec. 1.2.5], $p$ has no roots in $\mathcal{X}^{+}$if and only if $p(\partial) y=u$ is uniquely solvable over $\mathcal{O}_{+}^{\prime}$. By [4, Thm. 1 , sec. 1.2.5], this is equivalent to the condition that $p(\imath \zeta)^{-1} \in \mathcal{F}\left(\mathcal{O}_{+}^{\prime}\right)=\mathcal{L}^{+}$.

Clearly all the claims of Lemma 5.3 can be "time-reversed" to give corresponding results for $\mathcal{L}^{-}, \mathcal{S}_{-}, \mathcal{X}^{-}$, etc.

Claims 4 and 5 of Lemma 5.3 are our first input/output stability results. Claim 4, for example, states that if the input is both spatially and temporally rapidly decreasing (the first condition being a reasonable prior assumption on physical signals and the second meaning that it is "stable") and has zero past, then there exists a causal response with the same properties if and only if a certain condition (in fact CV) holds on $p$. Moreover, by time-autonomy (which can be assumed a priori), there cannot be any different causal system response; i.e., all causal responses are stable. Our main goal in what follows will be to generalize this result to the general system case.

5.3. Ideal convexity. In order to generalize the results in the previous section to systems, we will need certain properties of polynomials with respect to the set $\mathcal{X}^{+}$ to extend to ideals. 
Definition 5.4. We call a set $S \subseteq \mathbb{C}^{n}$ codimension $k$-convex, $k=1, \ldots, n$, if for any codimension $k$ prime ideal $J$ we have

$$
\mathcal{V}(J) \cap S=\emptyset \Rightarrow \exists f \in J: \mathcal{V}(f) \cap S=\emptyset .
$$

We say that $S$ is ideal-convex if property (28) holds for any (not necessarily prime) ideal $J$.

The first of these properties was introduced in [32], in which it is shown that the closed unit polydisc is codimension $k$-convex for all $k$. Codimension 1 -convexity is trivial, since any prime ideal of codimension 1 in $\mathbb{C}[s]$ is principal (it must contain an irreducible polynomial and so must be equal to the codimension 1 prime ideal generated by that polynomial). It was also observed in [32] that if $\mathcal{S}$ is codimension $k$-convex for $k=1, \ldots, n$, then $S$ is ideal convex; this is essentially due to claim 2 of the following simple but important result.

Theorem 5.5. Let $S \subseteq \mathbb{C}^{n}$ be one of the sets: $\imath \mathbb{R}^{n}, \mathcal{X}^{+}, \mathcal{X}^{-}$. We have

1. $S$ is codimension $n$-convex.

2. If the minimal prime divisors of an ideal satisfy (28), then the ideal itself also does.

3. For $n=2, S$ is ideal-convex.

Proof.

1. Suppose that $J$ is a maximal ideal with variety not intersecting $\mathcal{X}^{+}$. Both real and complex coefficient polynomial rings are covered by the following argument. Let $\left(\alpha_{1}, \ldots, \alpha_{n-1}, \beta\right)$ be a point in $\mathcal{V}(J)$. Then either $\Re\left(\alpha_{i}\right) \neq 0$ for some $i=1, \ldots, n-1$, or else $\Re(\beta)<0$. In the first case, $f:=\left(s_{i}-\alpha_{i}\right)\left(s_{i}-\overline{\alpha_{i}}\right)$ is a suitable polynomial in $J$, where - denotes complex conjugate. In the second case, $f:=\left(s_{n}-\beta\right)\left(s_{n}-\bar{\beta}\right)$ may be chosen. The argument for $\mathcal{X}^{-}$is symmetric and that for $\imath \mathbb{R}^{n}$ similar but simpler.

2. This property holds for arbitrary $S$. For suppose that $I$ is a given ideal such that $\mathcal{V}(I) \cap S=\emptyset$, and that $J_{1}, \ldots, J_{l}$ are prime divisors of $I$ and satisfy property (28). Then the product $f$ of the corresponding polynomials $f_{i} \in J_{i}$ is contained in the intersection of the $J_{i}$, which equals the radical of $I$, and therefore some power $f^{k}$ of $f$ is in $I$, and we have $\mathcal{V}\left(f^{k}\right) \cap S=\emptyset$ as required.

3 . For $n=2$, any nonzero prime ideal has codimension either 1 or $n$; using claim 1 we have that property (28) holds for all prime ideals. By claim $2, S$ is ideal-convex.

An important open question is whether the sets $S$ in the preceding theorem are actually ideal-convex for all $n$. We will see in section 7 that this question has major implications for input/output stability. Ideal-convexity can also be expressed (see also Proposition 1 in [28]) as a "(weak) Nullstellensatz"-type result for the localized ring $U^{-1} \mathbb{C}[s]$, where $U$ is the multiplicatively closed set of all polynomials which do not vanish anywhere in the given domain.

In [33], Valcher characterizes stability of autonomous behaviors for "square" behaviors (those admitting a square kernel representation matrix) and finite-dimensional behaviors (those for which the corresponding system module has codimension $n$ ). These seem to correspond to principal modules/ideals and maximal ideals, respectively, and we suspect that the generalization of [33] to the $n D$ case hits a problem analogous to proving ideal-convexity here.

6. Structure theory over $\mathcal{S}, \mathcal{S}^{\prime}, \mathcal{S}_{+}, \mathcal{S}_{\oplus}^{\prime}$. In this section, we will develop some basic but highly nontrivial results concerning the structure of behaviors over $\mathcal{S}$ and $\mathcal{S}^{\prime}$, and then $\mathcal{S}_{+}$and $\mathcal{S}_{\oplus}^{\prime}$. The second set of results and proofs follow the structure 
of the first. The results for $\mathcal{S}$ and $\mathcal{S}^{\prime}$ are also included for independent interest. Many of the results in this section and section 7 are restricted to the case $n=2$; this is due only to the lack of a proof that the sets $S$ in Theorem 5.5 are ideal-convex for all $n$. The material in this section and section 7 is entirely new, barring Theorem 6.1.

We begin by recalling a result on (Willems) closure from [36], which, however, we state in the most general form as indicated in that paper.

TheOREM 6.1. Let $\mathcal{W}$ be a module over $\mathbb{C}[s]$ and $\mathcal{N}$ a submodule of $\mathbb{C}[s]^{1 \times q}$ for some q. Suppose that $\mathcal{N}=\cap_{i=1}^{t} \mathcal{N}_{i}$ is an irredundant decomposition of $\mathcal{N}$ in $\mathbb{C}[s]^{1 \times q}$, where $\mathcal{N}_{i}$ is a $J_{i}$-primary submodule of $\mathbb{C}[s]^{1 \times q}$ for some prime ideals $J_{1}, \ldots, J_{t}$. Let the components be ordered so that for some $r \in 0, \ldots, t, J_{1}, \ldots, J_{r}$ each annihilate some nonzero element of $\mathcal{W}$, but $J_{r+1}, \ldots, J_{t}$ do not. Assume that each of the primes $J_{i}, i=1, \ldots, r$, is contained in some prime $K_{i}$ which also annihilates some nonzero element of $\mathcal{W}$, and for which

$$
\left(0: K_{i}^{\infty}\right)_{\mathcal{W}}:=\left\{w \in \mathcal{W} \mid K_{i}^{l} w=0 \text { for some } l \in \mathcal{N}\right\}
$$

is an injective $\mathbb{C}[s]$-module. Then the closure of $\mathcal{N}$ with respect to $\mathcal{W}$ is given by

$$
\mathcal{N}^{\perp \perp}=\bigcap_{i=1}^{r} \mathcal{N}_{i} .
$$

The assumption holds in particular whenever $\mathcal{W}$ is itself injective.

Proof. This result is a small refinement of [36, Thm. 3.9], as indicated in the comments following that result. For completeness, we sketch the necessary modifications to the proof given in [36] to achieve this refinement.

As shown in the original proof, the inclusion " $\supseteq$ " in (29) does not require any assumption on the primes $J_{1}, \ldots, J_{r}$. For the reverse inclusion, set $\mathcal{D}=\mathbb{C}[s], \mathcal{M}=$ $\mathcal{D}^{1 \times q} / \mathcal{N}$, and $\mathcal{L}=\cap_{i=1}^{r} \mathcal{M}_{i}, \mathcal{M}_{i}=\mathcal{N}_{i} / \mathcal{N}$, as in the original proof. As in the original proof, if $x \in \mathcal{M} \backslash \mathcal{L}$, then there exist $i \in 1, \ldots, r$ and $a \in \mathcal{D}$ with $\mathcal{D}\left(a x+\mathcal{M}_{i}\right) \cong \mathcal{D} / J_{i}$. Now by assumption, $J_{i}$ is contained in $K_{i}$ with $\left(0: K_{i}^{\infty}\right)_{\mathcal{W}}$ injective and also nonzero as $K_{i}$ annihilates some nonzero element of $\mathcal{W}$. As in the argument for $P_{j}$ in the original proof, $K_{i}$ must be contained in some prime $Q$ with a copy of the injective hull $E(\mathcal{D} / Q)$ of $\mathcal{D} / Q$ embedded in $\left(0: K_{i}^{\infty}\right)_{\mathcal{W}}$. We therefore have a sequence of maps of the form

$$
\mathcal{D}\left(a x+\mathcal{M}_{i}\right) \stackrel{\cong}{\rightrightarrows} \mathcal{D} / J_{i} \stackrel{\rho_{1}}{\rightarrow} \mathcal{D} / K_{i} \stackrel{\rho_{2}}{\rightarrow} \mathcal{D} / Q \rightarrow E(\mathcal{D} / Q) \rightarrow\left(0: K_{i}^{\infty}\right)_{\mathcal{W}},
$$

where $\rho_{1}$ and $\rho_{2}$ are the natural projections. Call the composition map $w_{1}$. As in the original proof, if $w_{1}\left(a x+\mathcal{M}_{i}\right)=0$, then we must have $\left(\rho_{2} \circ \rho_{2}\right)\left(1+J_{i}\right)=0$ in $\mathcal{D} / Q$, which entails that $1 \in Q$. This is impossible, so $w_{1}\left(a x+\mathcal{M}_{i}\right) \neq 0$ and $w_{1}$ is not the zero map. As in the original proof, $w_{1}$ can then be extended using injectivity of $\left(0: K_{i}^{\infty}\right)_{\mathcal{W}}$ to a nonzero map $w: \mathcal{M} \rightarrow \mathcal{W}$, which further is nonzero at $x$. This is enough to complete the proof, as explained in [36].

The final claim follows since injectivity of $\mathcal{W}$ guarantees injectivity of $\left(0: I^{\infty}\right)_{\mathcal{W}}$ for any ideal $I[14]$.

6.1. Structure theory over $\mathcal{S}, \mathcal{S}^{\prime}$. In particular, we may derive from Theorem 6.1 the characterization by Shankar of closure with respect to $\mathcal{S}^{\prime}$ [29] (this latter result, however, preceded and motivated the theorem above). $\mathcal{S}^{\prime}$ is injective, as proved originally by Malgrange [12] and more recently by Shankar [31], and therefore 
Theorem 6.1 applies; a prime $J$ kills some nonzero element of $\mathcal{S}^{\prime}$ if and only if it vanishes at some imaginary point. We repeat Shankar's result from [29] here, together with an alternative form for a special case.

Corollary 6.2. Suppose that $\mathcal{N}=\cap_{i=1}^{t} \mathcal{N}_{i}$ is an irredundant decomposition of a submodule $\mathcal{N}$ of $\mathbb{C}[s]^{1 \times q}$, where $\mathcal{N}_{i}$ is $J_{i}$-primary. Let the components be ordered so that for some $r \in 0, \ldots, t, J_{1}, \ldots, J_{r}$ each vanish at some point $\imath \mathbb{R}^{n}$ but $J_{r+1}, \ldots, J_{t}$ do not. Then the closure of $\mathcal{N}$ with respect to $\mathcal{W}$ is equal to $\cap_{i=1}^{r} \mathcal{N}_{i}$. Moreover, in the case where the primes are minimal in the set $J_{r+1}, \ldots, J_{t}$ and each has codimension 1 or $n$ (e.g., when $n \leq 2)$, there exists a polynomial $f$ with no imaginary roots such that the closure with respect to $\mathcal{W}$ may be written

$$
\mathcal{N}^{\perp \perp}=\left\{v \in \mathbb{C}[s]^{1 \times q} \mid \exists k \in \mathbb{N}, f^{k} v \in \mathcal{N}\right\} .
$$

Proof. The equality

$$
\mathcal{N}^{\perp \perp}=\bigcap_{i=1}^{r} \mathcal{N}_{i}
$$

is already given [29]. Now suppose that the primes are minimal among $J_{r+1}, \ldots, J_{t}$ and each has codimension 1 or $n$. By Theorem 5.5 there then exists $f \in \cap_{i=r+1}^{t} J_{i}$ with no imaginary roots. Note that $f$ is contained in $J_{r+1}, \ldots, J_{t}$ but not in any of $J_{1}, \ldots, J_{r}$ (since these do have imaginary roots). Hence by [3, Prop. 3.13], the module

$$
H_{(f)}^{0}(\mathcal{M}):=\left\{v+\mathcal{N} \in \mathcal{M} \mid f^{k} v \in \mathcal{N} \text { for some } k \in \mathcal{N}\right\}
$$

agrees with $\cap_{i=1}^{r} \mathcal{N}_{i} / \mathcal{N}$, where $\mathcal{M}=\mathbb{C}[s]^{1 \times q} / \mathcal{N}$. This together with (31) gives us the required result.

Note from the proof that the assumptions in the second claim of the corollary may be dropped if it can be shown that $\imath \mathbb{R}^{n}$ is ideal-convex for any $n$. From the corollary we can give conditions for one behavior over $\mathcal{S}^{\prime}$ to be contained in another (when the assumptions of Corollary 6.2 hold).

CoROLlaRY 6.3. Let $\mathcal{B}_{1}=\operatorname{ker}_{\mathcal{S}^{\prime}} R_{1}$ and $\mathcal{B}_{2}=\operatorname{ker}_{\mathcal{S}^{\prime}} R_{2}$ be two behaviors contained in $\left(\mathcal{S}^{\prime}\right)^{q}$ for some q. Let $\mathcal{N}_{1}$ be the row span of $R_{1}$ over $\mathbb{C}[s]$. Suppose that the primes, minimal among those associated primes of the module $\mathbb{C}[s]^{1 \times q} / \mathcal{N}_{1}$ with no imaginary points in their varieties, each have codimension 1 or $n$; this occurs, in particular, when $n \leq 2$. Then $\mathcal{B}_{1} \subseteq \mathcal{B}_{2}$ if and only if there exist a polynomial $f$ with no imaginary roots and a polynomial matrix $L$, such that $f R_{2}=L R_{1}$.

Proof. Suppose that such an $f$ and $L$ exist with $f R_{2}=L R_{1}$. Then for any $w \in \operatorname{ker}_{\mathcal{S}^{\prime}} R_{1}, f(\partial)\left(R_{2}(\partial) w\right)=0$. Since $f$ has no imaginary roots, by Corollary 6.2 the closure with respect to $\mathcal{S}^{\prime}$ of the ideal generated by $f$ is equal to $\mathbb{C}[s]$; i.e., $f$ kills no nonzero element of $\mathcal{S}^{\prime}$. Hence $R_{2}(\partial) w=0$ as required.

Conversely, suppose that $\mathcal{B}_{1} \subseteq \mathcal{B}_{2}$. Writing $\mathcal{N}_{1}$ and $\mathcal{N}_{2}$ for the row spans of $R_{1}$ and $R_{2}$, respectively, we have

$$
\mathcal{N}_{2} \subseteq \mathcal{N}_{2}^{\perp \perp} \subseteq \mathcal{N}_{1}^{\perp \perp}
$$

where ${ }^{\perp \perp}$ denotes closure with respect to $\mathcal{S}^{\prime}$. By the second claim of Corollary 6.2 (which requires the given assumptions on $\mathbb{C}[s]^{1 \times q} / \mathcal{N}_{1}$ or on $n$ ), there is a polynomial $f$ with no imaginary roots such that for each row of $R_{2}, f^{k}$ times that row is in $\mathcal{N}_{1}$ for sufficiently large $k$. Note that $f^{k}$ also has no imaginary roots. The converse follows. 
Again, the assumptions in Corollary 6.3 are needed only because it has not been proven yet that $\imath \mathbb{R}^{n}$ is ideal-convex. These assumptions prove an obstacle to the next result, which for this reason only is restricted to the special cases when $P$ is a polynomial or $n \leq 2$.

THEOREM 6.4. Let

$$
\mathcal{B}:=\left\{(u, y) \in\left(\mathcal{D}^{\prime}\right)^{m+p} \mid P(\partial) y=Q(\partial) u\right\}
$$

be a behavior with the indicated input/output structure and corresponding transfer matrix $G$. Suppose that either $P$ is a single polynomial or $n \leq 2$. Then the following are equivalent:

1. The variables $u$ are free over $\mathcal{S}$ in $\mathcal{B}$.

2. $\operatorname{ker}_{\mathcal{S}^{\prime}} P^{*} \subseteq \operatorname{ker}_{\mathcal{S}^{\prime}} Q^{*}$.

3. There exist a polynomial $r$ with no imaginary roots and a polynomial matrix $L$, such that $G=\frac{1}{r} L$.

4. $\mathcal{B}$ has no imaginary controllable poles.

5. $G(\imath \zeta) \in \mathcal{L}^{p \times m}$.

Proof. $5 \Rightarrow 1$ : Suppose $G^{*}(\imath \zeta) \in \mathcal{L}^{p \times m}$. Then for any $u \in \mathcal{S}^{m}$ we have that $v(\zeta):=G^{*}(\imath \zeta) \hat{u}(\zeta) \in \mathcal{S}^{p}$, where $\hat{\imath}$ denotes the Fourier transform, using claim 2 of Lemma 5.2. Now $P(\imath \zeta) v(\zeta)=Q(\imath \zeta) \hat{u}(\zeta)$, so the inverse Fourier transform of $v$ is a solution $y$ to $P(\partial) y=Q(\partial) u$.

$1 \Rightarrow 2$ : If the variables $u$ are free over $\mathcal{S}$, then $\operatorname{im}_{\mathcal{S}} Q \subseteq \operatorname{im}_{\mathcal{S}} P$. Dualizing (in the distributional sense), we have condition 2 .

$2 \Rightarrow 3$ : Suppose that condition 2 holds. If $P$ is a single polynomial, then the associated primes of the system module of $P^{*}(\partial)$ all have codimension 1 ; thus in either this case or when $n \leq 2$, the conditions of Corollary 6.3 hold. Thus by this corollary, there exist a polynomial $r$ with no imaginary roots and a polynomial matrix $L$, with $r^{*} Q^{*}=L^{*} P^{*}$, and hence $P(L / r)=Q$. Since $P$ has full column rank, $L / r$ equals $G$.

$3 \Rightarrow 4$ : This is immediate from Lemma 2.3.

$4 \Rightarrow 5$ : Suppose $\mathcal{B}$ has no imaginary controllable poles, and let $r$ be the least common denominator of $G$. So, in particular, $G=(1 / r) L$ for some polynomial matrix $L$, and $r$ has no imaginary zeros. By claim 5 of Lemma $5.2,(1 / r) I_{p} \in \mathcal{L}^{p \times p}$, and now by claim 1 of Lemma $5.2, G=(1 / r) L \in \mathcal{L}^{p \times m}$.

Similarly, we can also produce the following lemma.

LEMma 6.5. With the notation of Theorem 6.4 (but no assumptions on $n$ or $P$ are needed), the following are equivalent:

1. The variables $u$ are free over $\mathcal{C}_{0}^{\infty}$ in $\mathcal{B}$.

2. The variables $u$ are free over $\mathcal{E}^{\prime}$ in $\mathcal{B}$.

3. $\mathcal{B}$ has no controllable poles or, equivalently, the outputs are observable from the inputs in $\mathcal{B}^{c}$.

4. $G$ is a polynomial matrix.

Proof. For either case $\mathcal{C}_{0}^{\infty}, \mathcal{E}^{\prime}$, we use the same proof structure as for Theorem 6.4; dualizing condition 1 or 2 gives a condition for inclusion of behaviors over $\mathcal{D}^{\prime}$ or over $\mathcal{C}^{\infty}$, which results in $G$ being a polynomial matrix. Conversely, if $G$ is a polynomial matrix, then with input $u \in\left(\mathcal{C}_{0}^{\infty}\right)^{m}$ or $u \in\left(\mathcal{E}^{\prime}\right)^{m}$ we can simply choose $y=G(\partial) u$.

For completeness, we offer a result without prior assumption of an input/output structure. 
Corollary 6.6. Let a behavior $\mathcal{B}$ be given by

$$
\mathcal{B}:=\left\{(u, y) \in\left(\mathcal{D}^{\prime}\right)^{m+p} \mid P(\partial) y=Q(\partial) u\right\},
$$

where $(u, y)$ is an arbitrary partition of the system variables. Then the following hold:

1. The variables $u$ are free over either $\mathcal{C}_{0}^{\infty}$ or $\mathcal{E}^{\prime}$ in $\mathcal{B}$ if and only if there exists a polynomial matrix $L$ with $P L=Q$. The maximum number of free variables over $\mathcal{C}_{0}^{\infty}$ or $\mathcal{E}^{\prime}$ of a behavior $\mathcal{B}$ with kernel representation $R$ equals the number of systems variables minus the minimum number of columns of $R$ needed to generate column span over $\mathbb{C}[s]$.

2. Suppose $n \leq 2$. The variables $u$ are free over $\mathcal{S}$ if and only if there exist a polynomial $r$ and a polynomial matrix $L$, such that $P L=Q r$. The maximum number of free variables over $\mathcal{S}$ of a behavior $\mathcal{B}$ with kernel representation $R$ equals the number of system variables minus the number of columns of $R$ needed to generate the column span over the localized ring $U^{-1} \mathbb{C}[s]$, where $U$ is the set of polynomials with no imaginary roots.

Proof. We prove the second claim only, that $u$ is free over $\mathcal{S}$ if and only if given that $L, r$ exist follows entirely from the arguments $1 \Rightarrow 2 \Rightarrow 3,5 \Rightarrow 1$, which do not require prior existence of an input/output structure. Finally, note that a given set of columns forming a submatrix $P$ of $R$ generates the column span of $R$ over $U^{-1} \mathbb{C}[s]$ if and only if there exists a matrix $K$ over $U^{-1} \mathbb{C}[s]$ with $P K=Q$, where $Q$ consists of the complementary columns of $R$. This is clearly equivalent to the given condition for freeness over $\mathcal{S}$ of the variables corresponding to the columns of $Q$; the result follows.

Recall that over $\mathcal{C}^{\infty}, \mathcal{D}^{\prime}$, or $\mathcal{S}^{\prime}$, a set of variables are free if and only if the corresponding elements in the system module are linearly independent over $\mathbb{C}[s][35$, Lem. 5.3]; this is due to injectivity of these three spaces. Equivalently, with the notation of Corollary 6.6, there exists a rational function matrix $G$ with $P G=Q$. For $n \leq 2$, the corollary above completes the description of free variables over the classical spaces; the full generalization to $n>2$ for $\mathcal{S}$ rests on proving ideal-convexity of $\imath \mathbb{R}^{n}$.

6.2. Structure theory over $\mathcal{S}_{+}, \mathcal{S}_{\oplus}^{\prime}$. Our next aim is to repeat the pattern of Corollaries 6.2 and 6.3 and Theorem 6.4, but for $\mathcal{S}_{\oplus}^{\prime}$ and $\mathcal{S}_{+}$instead of $\mathcal{S}^{\prime}$ and $\mathcal{S}$. This will give us results which we can then interpret in terms of stability. Notice that $\mathcal{S}_{\oplus}^{\prime}:=\mathcal{S}^{\prime} / \mathcal{S}_{-}$may be interpreted as the restriction of tempered distributions to $\mathbb{R}_{+}^{n}$.

To begin, we demonstrate that usual results on adjoint operators apply to the space $\mathcal{S}_{+}$and dual $\mathcal{S}_{\oplus}^{\prime}$.

Lemma 6.7. If $R \in \mathbb{C}[s]^{g \times q}$, then for any $f \in\left(\mathcal{S}_{\oplus}^{\prime}\right)^{q}, \phi \in\left(\mathcal{S}_{+}\right)^{g}$ we have

$$
\langle R(\partial) f, \phi\rangle=\left\langle f, R^{*}(\delta) \phi\right\rangle .
$$

Hence $f \in \operatorname{ker}_{\mathcal{S}_{\oplus}}^{\prime} R$ if and only if $f$ kills $\operatorname{im}_{\mathcal{S}_{+}} R^{*}$.

Proof. Let $R, f$, and $\phi$ be as given; denote by $\iota$ the natural inclusion $\mathcal{S}_{+} \rightarrow \mathcal{S}$. Let $\bar{f} \in\left(\mathcal{S}^{\prime}\right)^{q}$ be some element such that its equivalence class in $\mathcal{S}_{+}^{\prime}$ agrees with $f$, and let $\bar{h}=R(\partial) \bar{f} \in\left(\mathcal{S}^{\prime}\right)^{g}$; then $\bar{h}+\left(\mathcal{S}_{+}^{\prime}\right)^{g}=R(\partial) f$ by definition of differentiation on a factor space such as $\mathcal{S}_{\oplus}^{\prime}$. We now have

$$
\begin{aligned}
\langle R(\partial) f, \phi\rangle & :=\langle\bar{h}, \iota(\phi)\rangle \\
& =\langle R(\partial) \bar{f}, \iota(\phi)\rangle \\
& =\left\langle\bar{f}, R^{*}(\partial) \iota(\phi)\right\rangle \\
& =\left\langle\bar{f}, \iota\left(R^{*}(\partial) \phi\right)\right\rangle \\
& =\left\langle f, R^{*}(\partial) \phi\right\rangle .
\end{aligned}
$$

The second claim is immediate. 
We now provide some elementary results on the structure of $\mathcal{S}_{\oplus}^{\prime}$ as a differential module.

Lemma 6.8. Let $r$ be a polynomial. Then the differential operator $r(\partial)$ kills some nonzero element of $\mathcal{S}_{\oplus}^{\prime}$ if and only if $r$ has a root in $\mathcal{X}^{-}$.

Proof. Suppose that $r$ has no roots in $\mathcal{X}^{-}$and let $\bar{w}=w+\mathcal{S}_{-}^{\prime} \in \mathcal{S}_{\oplus}^{\prime}$ be such that $r(\partial) \bar{w}=0$. Then $r(\partial) w \in \mathcal{S}_{-}^{\prime}$, so $r(\imath \zeta) \hat{w}(\zeta) \in \mathcal{F}\left(\mathcal{S}_{-}^{\prime}\right)$, where $\mathcal{F}$ denotes Fourier transform, and by claim 3 of Lemma 5.3 this space equals $\mathcal{L}^{-}$. By claim 6 of Lemma 5.3, $1 / r(\imath \zeta) \in \mathcal{L}^{-}$, and so by claim 1 of Lemma $5.3, \hat{w}(\zeta) \in \mathcal{L}^{-}=\mathcal{F}\left(\mathcal{S}_{-}^{\prime}\right)$ also. Hence $\bar{w}=0$.

Conversely, suppose that $r$ does not have a root $\zeta \in\left(\imath \mathbb{R}^{n-1} \times \overline{\mathbb{C}_{-}}\right)$. Corresponding to this is a nonzero exponential trajectory $u$ of frequency $\zeta$ which lies in $\mathcal{C}^{\infty}$. Multiplying it by a suitable "cut-off" function, we have a trajectory $w \in L^{\infty} \subseteq \mathcal{S}^{\prime}$ which agrees with $u$ on $\mathbb{R}_{+}^{n}$. As $u$ is killed by $r(\partial)$, the support of $r(\partial) w$ lies in $\mathbb{R}_{-}^{n}$, and therefore $r(\partial)\left(w+\mathcal{S}_{-}^{\prime}\right)=0$, whereas $w+\mathcal{S}_{-}^{+} \neq 0$ because $u$ is nonzero on $\mathbb{R}_{+}^{n}$.

Next we generalize this to ideals, which requires ideal-convexity, and so is restricted to special cases, e.g., $n \leq 2$.

LEMma 6.9. If an ideal I kills some nonzero element of $\mathcal{S}_{\oplus}^{\prime}$, then $\mathcal{V}(I) \cap \mathcal{X}^{-} \neq \emptyset$. The converse holds when the minimal primes containing I each have codimension 1 or $n$ (e.g., when $n \leq 2)$.

Proof. Suppose that $I$ vanishes at some point $\zeta \in\left(\imath \mathbb{R}^{n-1} \times \overline{\mathbb{C}_{-}}\right)$. As in the proof of Lemma 6.8, we can construct a nonzero trajectory $w \in \mathcal{S}_{\oplus}^{\prime}$ which is killed by the maximal ideal corresponding to $\zeta$, and therefore killed by $I$.

Conversely, suppose that $\mathcal{V}(I) \cap \mathcal{X}^{-}=\emptyset$. By Theorem 5.5 (using the assumption on $I$ ), there exists $f \in I$ which has no roots in $\imath \mathbb{R}^{n-1} \times \overline{\mathbb{C}_{-}}$. Now if $I w=0$ for some $w \in \mathcal{S}_{\oplus}^{\prime}$, then $f(\partial) w=0$ in particular, so by Lemma $6.8, w=0$.

Lemmas 6.8 and 6.9 are basic results on closure with respect to $\mathcal{S}_{\oplus}^{\prime}$. In order to extend them to more general cases, we need some injectivity properties of certain modules associated to $\mathcal{S}_{\oplus}^{\prime}$.

Lemma 6.10. For any maximal ideal $I,\left(0: I^{\infty}\right)_{\mathcal{S}_{\oplus}^{\prime}}$ is injective.

Proof. In the case where $I$ annihilates no nonzero element of $\mathcal{S}_{\oplus}^{\prime},\left(0: I^{\infty}\right)_{\mathcal{S}_{\oplus}^{\prime}}=0$ is trivially injective. So suppose that $I$ does annihilate some nonzero element of $\mathcal{S}_{\oplus}^{\prime}$, which by Lemma 6.9 means that $\exists \zeta \in \mathcal{V}(I) \cap \mathcal{X}^{-}$; indeed we must have $\mathcal{V}(I)=\{\zeta\}$.

Now let $\Psi:\left(0: I^{\infty}\right)_{\mathcal{D}^{\prime}} \rightarrow\left(0: I^{\infty}\right)_{\mathcal{D}_{\oplus}^{\prime}}$ be the module homomorphism induced by the natural projection $\mathcal{D}^{\prime} \rightarrow \mathcal{D}_{\oplus}^{\prime}$. Since $\left(0: I^{\infty}\right)_{\mathcal{D}^{\prime}}=\left(0: I^{\infty}\right)_{\mathcal{C}^{\infty}}$ is the set of polynomial exponentials with frequency $\zeta[18,37], \Psi$ is clearly injective. Consider an arbitrary element $w \in\left(0: I^{\infty}\right)_{\mathcal{D}^{\prime}}$. We can write $w=p w^{\prime}$, where $p$ is a polynomial function and $w^{\prime}$ is an exponential trajectory of frequency $\zeta$. Letting $f \in \mathcal{C}^{\infty}(\mathbb{R})$ be a "cut-off" function with $f(t)=1$ for $t \geq 0$ and $f(t)=0$ for $t \ll 0$, by the location of $\zeta$ we have that $f(t) w^{\prime}(x, t) \in L^{\infty} \subseteq \mathcal{S}^{\prime}$. Since by the Fourier transformation $\mathcal{S}^{\prime}$ is closed under multiplication by polynomials, $f w=p f w^{\prime} \in \mathcal{S}^{\prime}$ also. Now $f w$ agrees with $w$ on $\mathbb{R}_{+}^{n}$, so $\Phi(w)=\Phi(f w)=f w+\mathcal{D}_{-}^{\prime} \in \mathcal{D}_{\oplus}^{\prime}$. It follows that

$$
\operatorname{im} \Psi \subseteq \frac{\mathcal{S}^{\prime}+\mathcal{D}_{-}^{\prime}}{\mathcal{D}_{-}^{\prime}} \cong \frac{\mathcal{S}^{\prime}}{\mathcal{S}^{\prime} \cap \mathcal{D}_{-}^{\prime}}=\mathcal{S}_{\oplus}^{\prime} .
$$

Hence we can construct another injective module homomorphism $\Phi:\left(0: I^{\infty}\right)_{\mathcal{D}^{\prime}} \rightarrow(0$ : $\left.I^{\infty}\right)_{\mathcal{S}_{\oplus}^{\prime}}$. The module $\left(0: I^{\infty}\right)_{\mathcal{D}^{\prime}}$ is injective because $\mathcal{D}^{\prime}$ is injective [14], so it remains to show that $\Phi$ is surjective, or equivalently that the image of $\Psi$ is $\left(0: I^{\infty}\right)_{\mathcal{W}}$, where $\mathcal{W}=\left(\mathcal{S}^{\prime}+\mathcal{D}_{-}^{\prime}\right) / \mathcal{D}^{\prime}$. However, if $\bar{w}=w+\mathcal{D}^{\prime}$ with $w \in \mathcal{S}^{\prime}$ and $I^{k} \bar{w}=0$ for some $k$, we have that the support of $I^{k} w$ is in $\mathbb{R}_{-}^{n}$; i.e., $w$ is a solution in $\mathbb{R}_{+}^{n}$ to the equations 
of $I^{k}$. However, any distributional solution in $\mathbb{R}_{+}^{n}$ to the equations of $I^{k}$ necessarily agrees in that region with a polynomial exponential of degree $k$, i.e., an element $\hat{w}$ of $\left(0: I^{k}\right)_{\mathcal{D}^{\prime}}$, as can be seen by induction on $k$ (the base case $k=1$ is clear as it reduces to the ODE case). Hence $\Psi(\hat{w})=w$ as required.

Lemma 6.10 leaves the important open question as to whether $\mathcal{S}_{\oplus}^{\prime}$ itself is an injective module. We now provide a technical result, which sets up the conditions necessary to apply Theorem 6.1 over $\mathcal{S}_{\oplus}^{\prime}$.

Corollary 6.11. Any prime ideal which vanishes at some point of $\mathcal{X}^{-}$is contained in a prime I which has the same property, and for which $\left(0: I^{\infty}\right)_{\mathcal{S}_{\oplus}^{\prime}}$ is injective.

Proof. Let $J$ be a prime for which $\mathcal{V}(J) \cap \mathcal{X}^{-} \neq \emptyset$. Thus there exists a maximal ideal $I$ which vanishes at some point of $\mathcal{X}^{-}$and for which $\mathcal{V}(I) \subseteq \mathcal{V}(J)$, so $J \subseteq I$. Lemma 6.10 now completes the proof.

In certain special cases, e.g., $n \leq 2$, we can now characterize closure over $\mathcal{S}_{\oplus}^{\prime}$.

Corollary 6.12. Suppose that $\mathcal{N}=\cap_{i=1}^{t} \mathcal{N}_{i}$ is an irredundant primary decomposition of a submodule $\mathcal{N}$ of $\mathbb{C}[s]^{1 \times q}$, where $\mathcal{N}_{i}$ is $J_{i}$-primary. Let the components be ordered so that for some $r \in 0, \ldots, t, J_{1}, \ldots, J_{r}$ each vanishes at some point of $\mathcal{X}^{-}$but $J_{r+1}, \ldots, J_{t}$ do not. Suppose further that the primes $J_{r+1}, \ldots, J_{t}$ each have codimension 1 or $n$ (e.g., $n \leq 2$ ). Then the closure of $\mathcal{N}$ with respect to $\mathcal{S}_{\oplus}^{\prime}$ is equal to $\cap_{i=1}^{r} \mathcal{N}_{i}$, and, furthermore, there exists a polynomial $f$ with no roots in $\mathcal{X}^{-}$such that the closure with respect to $\mathcal{S}_{\oplus}^{\prime}$ may be written

$$
\mathcal{N}^{\perp \perp}=\left\{v \in \mathbb{C}[s]^{1 \times q} \mid \exists k \in \mathbb{N}, f^{k} v \in \mathcal{N}\right\} .
$$

Proof. Suppose that the primes $J_{r+1}, \ldots, J_{t}$ each has codimension 1 or $n$. Then by Lemma $6.9, J_{1}, \ldots, J_{r}$ each annihilate a nonzero element of $\mathcal{S}_{\oplus}^{\prime}$, whereas $J_{r+1}, \ldots, J_{t}$ do not. Using also Corollary 6.11, we have that $\mathcal{N}^{\perp \perp}=\cap_{i=1}^{r} \mathcal{N}_{i}$ by Theorem 6.1. Now by Theorem 5.5 there exists $f \in \cap_{i=r+1}^{t} J_{i}$ with no roots in $\mathcal{X}^{-}$. As in the proof of Corollary 6.2, we can now establish (33).

Note that the codimension conditions in Corollary 6.12 in particular hold when $\mathcal{N}$ is equal to $\mathbb{C}[s] \cdot p(s)$ for $p$ a single polynomial.

COROLlaRY 6.13. Let $\mathcal{B}_{1}=\operatorname{ker}_{\mathcal{S}_{\oplus}^{\prime}} R_{1}$ and $\mathcal{B}_{2}=\operatorname{ker}_{\mathcal{S}_{\oplus}^{\prime}} R_{2}$ be two behaviors contained in $\left(\mathcal{S}_{\oplus}^{\prime}\right)^{q}$ for some $q$. Let $\mathcal{N}_{1}$ be the row span of $R_{1}$ over $\mathbb{C}[s]$. Suppose that those associated primes of $\mathbb{C}[s]^{1 \times q} / \mathcal{N}_{1}$ with varieties not intersecting $\mathcal{X}^{-}$each have codimension 1 or $n$ (for example, $\mathbb{C}[s]^{1 \times q} / \mathcal{N}_{1}$ is principal or $n \leq 2$ ). Then $\mathcal{B}_{1} \subseteq \mathcal{B}_{2}$ if and only if there exist a polynomial $f$ with no roots in $\mathcal{X}^{-}$and a polynomial matrix $L$, such that $f R_{2}=L R_{1}$.

Proof. The proof uses exactly the same argument as for Corollary 6.3.

It is the failure to prove ideal-convexity of $\mathcal{X}^{-}$which prevents generalization of Corollaries 6.12 and 6.13 to the general $n D$ case.

7. Stable input/output structures. Having finally done all the necessary groundwork, we can now consider input/output stability. We consider this only for input/output structures which are a priori causal (with respect to $\mathcal{C}^{\infty}$ or $\mathcal{D}^{\prime}$ according to the type of stability required); thus, in particular, we assume that $\mathcal{B}_{0, y}$ is timeautonomous.

DeFINITION 7.1. Let $\mathcal{B}$ be a behavior with associated input/output structure $(u, y)$, where $\mathcal{B}_{0, y}$ is time-autonomous and the input/output structure is causal. Call this input/output structure stable (with respect to $\mathcal{S}$ (resp., $\mathcal{S}^{\prime}$ )) if for any $u \in\left(\mathcal{S}_{+}\right)^{m}$ (resp., $u \in\left(\mathcal{S}_{+}\right)^{m}$ ) and $y \in\left(\mathcal{C}_{+}^{\infty}\right)^{p}$ (resp., $y \in\left(\mathcal{D}_{+}^{\prime}\right)^{p}$ ) for which $(u, y) \in \mathcal{B}$, we must have $y \in\left(\mathcal{S}_{+}\right)^{p}$ (resp., $\left.y \in\left(\mathcal{S}_{+}^{\prime}\right)^{p}\right)$. 
Thus, roughly speaking, an input/output structure is stable if any causal output response to a stable input is itself stable. Since it is reasonable to assume a priori that our input/output structure is causal, the existence of a $y \in\left(\mathcal{C}_{+}^{\infty}\right)^{p}$ corresponding to a $u \in\left(\mathcal{S}_{+}\right)^{m}$ is guaranteed. Moreover, if $\mathcal{B}_{0, y}$ is a priori time-autonomous, this $y$ is unique, and so in this case the input/output structure is stable with respect to $\mathcal{S}$ (resp., $\mathcal{S}^{\prime}$ ) if and only if the variables $u$ are free over the signal space $\mathcal{S}$ (resp., $\mathcal{S}^{\prime}$ ). Fortunately, using methods analogous to those in the proof of Theorem 6.4, we can now characterize freeness of variables over $\mathcal{S}_{+}$using the structure theory developed in the last section. The results for $\mathcal{S}_{+}$are, however, restricted to the special cases when $n \leq 2$ or $P$ is a single polynomial (i.e., there is a single system equation), due to the difficulty of proving ideal-convexity for $n>2$, whereas those for $\mathcal{S}_{+}^{\prime}$ give sufficient conditions for freeness only.

THEOREM 7.2. Let

$$
\mathcal{B}:=\left\{(u, y) \in\left(\mathcal{D}^{\prime}\right)^{m+p} \mid P(\partial) y=Q(\partial) u\right\}
$$

be a behavior with given input/output structure, and transfer matrix G. Suppose that either $P$ is a single polynomial or $n \leq 2$. Then the following are equivalent.

1. The variables $u$ are free over $\mathcal{S}_{+}$in $\mathcal{B}$.

2. $\operatorname{ker}_{\mathcal{S}_{\oplus}^{\prime}} P^{*} \subseteq \operatorname{ker}_{\mathcal{S}_{\oplus}^{\prime}} Q^{*}$.

3. There exist a polynomial $r$ with no roots in $\mathcal{X}^{+}$and a polynomial matrix $L$, such that $G=\frac{1}{r} L$.

4. $\mathcal{B}$ has no controllable poles in $\mathcal{X}^{+}$.

5. $G(\imath \zeta) \in\left(\mathcal{L}^{+}\right)^{p+m}$.

Proof. The proof has the same structure of that of Theorem 6.4.

$5 \Rightarrow 1$ : Suppose $G(\imath \zeta) \in\left(\mathcal{L}^{+}\right)^{p \times m}$. Then for any $u \in\left(\mathcal{S}_{+}\right)^{m}$ we have that $v(\zeta):=G(\imath \zeta) \hat{u}(\zeta) \in\left(\mathcal{S}^{+}\right)^{p}$, where $\hat{\bullet}$ denotes the Fourier transform, using claims 2 and 3 of Lemma 5.3. Now using claims 2 and 6 of Lemma 5.3, we have that $P(\imath \zeta) v(\zeta)=$ $Q(\imath \zeta) \hat{u}(\zeta)$, so the inverse Fourier transform of $v$ is a solution $y$ to $P(\partial) y=Q(\partial) u$. By claim 3 of Lemma 5.3, $y \in\left(\mathcal{S}_{+}\right)^{p}$.

$1 \Rightarrow 2$ : Suppose the variables $u$ are free over $\mathcal{S}_{+}$. Then $\operatorname{im}_{\mathcal{S}_{+}} Q \subseteq \operatorname{im}_{\mathcal{S}_{+}} P$. Using Lemma 6.7, we find the dual condition 2 .

$2 \Rightarrow 3$ : Suppose that condition 2 holds. If $P$ is a single polynomial, then the associated primes of the system module of $P^{*}(\partial)$ all have codimension 1 ; thus in either this case or when $n \leq 2$, the conditions of Corollary 6.13 hold. Thus by this corollary there exist a polynomial $r^{*}$ with no roots in $\mathcal{X}^{-}$and a polynomial matrix $L^{*}$, with $r^{*} Q^{*}=L^{*} P^{*}$. Hence $P(L / r)=Q$, where $r$ has no roots in $\mathcal{X}^{+}$. Since $P$ has full column rank, $L / r$ equals $G$.

$3 \Rightarrow 4$ : This part of the proof is again immediate from the fact that the controllable pole variety of $\mathcal{B}$ is the variety of the least common denominator of $G$.

$4 \Rightarrow 5$ : Suppose $\mathcal{B}$ has no controllable poles in $\mathcal{X}^{+}$, and let $r$ be the least common denominator of $G$, so in particular $G=(1 / r) L$ for some polynomial matrix $L$, and $r$ has no zeros in $\mathcal{X}^{+}$. By claim 6 of Lemma $5.3,(1 / r) I_{p} \in(\mathcal{L})^{p \times p}$, and now by claim 1 of Lemma $5.3, G=(1 / r) L \in\left(\mathcal{L}^{+}\right)^{p \times m}$.

Note. A topic for future research is to seek further characterizations for a principal module, other than computing its associated primes. This could well lead to generalizations of Theorems 6.4 and 7.2 with less restrictive assumptions.

Let us consider the case of single polynomials in the equivalent conditions $2 \equiv$ $3 \equiv 5$ in Theorem 7.2; take $q$ and $p \neq 0$ rather than their adjoints for ease of notation. We have that $\operatorname{ker}_{\mathcal{S}_{\oplus}^{\prime}} p \subseteq \operatorname{ker}_{\mathcal{S}_{\oplus}^{\prime}} q$ if and only if $p / \operatorname{gcd}(p, q)$ has no roots in $\mathcal{X}^{-}$if and 
only if $(q / p)(\imath \zeta) \in \mathcal{L}^{-}$. From this we may define an action of $\mathcal{L}^{-}$on $\mathcal{S}_{\oplus}^{\prime}$ : given any $u \in \mathcal{S}_{\oplus}^{\prime}$ we can choose an arbitrary $v \in \mathcal{S}_{\oplus}^{\prime}$ with $u=p(\partial) v$; this is possible as $\mathcal{S}_{\oplus}^{\prime}$ is a divisible $\mathbb{C}[s]$-module due to divisibility of $\mathcal{S}^{\prime}$. Now $y:=q(\partial) v \in \mathcal{S}_{\oplus^{\prime}}$ is uniquely determined by $u$, due to the condition $\operatorname{ker}_{\mathcal{S}_{\oplus}^{\prime}} p \subseteq \operatorname{ker}_{\mathcal{S}_{\oplus}^{\prime}} q$, and we have $p(\partial) y=q(\partial) u$. Thus we have an extension of the operator ring on $\mathcal{S}_{\oplus}^{\prime}$ from $\mathbb{C}[s]$ to $\mathcal{L}^{-}$, analogous to the construction of Glüsing-Lüerssen in [5] for delay-differential systems (however, in the current case, the extended ring is a localization, unlike in [5]). It may be profitable, as in the delay-differential case, to consider systems defined as equations over this extended ring. Analogous remarks apply to the signal space $\mathcal{S}^{\prime}$ and ring $\mathcal{L}$.

We now give a generalization of claim 5 of Lemma 5.3, which gives sufficient conditions for freeness of variables over $\mathcal{S}_{+}^{\prime}$. We suspect that these conditions are also necessary.

Corollary 7.3. Let

$$
\mathcal{B}:=\left\{(u, y) \in\left(\mathcal{D}^{\prime}\right)^{m+p} \mid P(\partial) y=Q(\partial) u\right\}
$$

be a behavior with given input/output structure and transfer matrix $G$, and suppose that the denominators of $G$ have no roots in $\mathbb{R}^{n-1} \times \mathbb{C}_{+}$. Then the variables $u$ are free over $\mathcal{S}_{+}^{\prime}$ in $\mathcal{B}$.

Proof. Let $\mathcal{B}, P, Q$, and $G$ be as given. Let $d$ be the least common denominator of $G$, so that $G d=N$, a polynomial matrix, and $d$ has no roots in $\mathbb{R}^{n-1} \times \mathbb{C}_{+}$. Let $u \in\left(\mathcal{S}_{+}^{\prime}\right)^{m}$ be arbitrary. Then by claim 5 of Lemma 5.3 , there exists $y \in\left(\mathcal{S}_{+}^{\prime}\right)^{p}$ satisfying

$$
d(\partial) y=N(\partial) u
$$

We now have that $P(\partial) d(\partial) y=P(\partial) N(\partial) u=Q(\partial) d(\partial) u$. Hence $d(\delta)$ kills $P(\partial) y-Q(\partial) u$. However, $d(\delta)$ can kill no elements of $\mathcal{S}_{+}^{\prime}$, again by claim 5 of Lemma 5.3, and so $P(\partial) y=Q(\partial) u$. This proves that the variables $u$ are free over $\mathcal{S}_{\oplus}^{\prime}$.

Note that the conditions of Corollary 7.3 are particularly met when the equivalent conditions of Theorem 7.2 are satisfied. One consequence of this corollary is that when $G$ is as specified, given any input $u$ which is a Dirac delta in one component and zero in the others (and so in $\left(\mathcal{S}_{+}^{\prime}\right)^{m}$ ), there is a corresponding causal output in $\left(\mathcal{S}_{+}^{\prime}\right)^{p}$. If we assume time-autonomy of $\mathcal{B}_{0, y}$, then these causal outputs are unique, and we may collect them into a matrix called the impulse response matrix $H_{i m p}$. When $G$ is further stable, the input-to-output map over $\mathcal{S}_{+}$, which exists due to Theorem 7.2, is then given by applying $H_{i m p}$ as a convolution operator. However, as shown in the proof $5 \Rightarrow 1$ of Theorem 7.2, it can also be given by Fourier transformation, multiplication by $G(\imath \zeta)$, and inverse Fourier transformation. Thus $H_{i m p}$ is indeed the inverse Fourier transform of the transfer matrix $G$. Moreover, by Lemma 5.3 we have $\mathcal{L}^{+}=\mathcal{F}\left(\mathcal{O}_{+}^{\prime}\right)$, so we have $H_{i m p} \in\left(\mathcal{O}_{+}^{\prime}\right)^{p \times m}$.

Our next result shows that, as for causality, when stability with respect to $\mathcal{S}$ of an input/output structure is defined, it is characterized purely in terms of the transfer matrix. This result is, however, restricted to the cases $n \leq 2$ or $P$ is a single polynomial. For the case of stability with respect to $\mathcal{S}^{\prime}$, no such restriction is needed, but only a sufficient condition is obtained.

THEOREM 7.4. Let $\mathcal{B}$ be a behavior with a given input/output structure $(u, y)$, such that $\mathcal{B}_{0, y}$ is time-autonomous, and associated transfer matrix $G$. If $G$ is weakly stable, then $(u, y)$ is causal with respect to $\mathcal{D}^{\prime}$ and stable with respect to $\mathcal{S}^{\prime}$. Moreover, suppose that either $n \leq 2$ or $\mathcal{B}_{0, y}$ is defined by a single polynomial. Then $(u, y)$ is 
both causal with respect to $\mathcal{C}^{\infty}$ and stable with respect to $\mathcal{S}$ if and only if $G$ is stable or, equivalently, if and only if $G(\imath \zeta) \in\left(\mathcal{L}^{+}\right)^{p \times m}$.

Proof. Let $\mathcal{B}$ be given with $\mathcal{B}_{0, y}$ time-autonomous. Suppose first that $G$ is weakly stable or stable (the equivalent characterization of stability of $G$ in terms of $\mathcal{L}^{+}$is immediate from Theorem 7.2). Let $d$ be the least common denominator of $G$, which satisfies the WCV condition and therefore obeys the Gårding condition (20). As $\mathcal{B}_{0, y}$ is time-autonomous, $\left(\mathcal{B}^{c}\right)_{0, y}$ is time-autonomous also by Corollary 4.5. Since $\mathcal{V}(d)$ is equal to the characteristic variety of $\left(\mathcal{B}^{c}\right)_{0, y}$ (Lemma 2.3$),(0,0, \ldots, 1)$ is also a noncharacteristic direction for $d(\partial)$; hence, being a single polynomial, $d$ is hyperbolic by Theorem 3.3. In other words, $G$ is causal. Now by Theorem 4.7, the input/output structure on $\mathcal{B}$ is causal with respect to both $\mathcal{C}^{\infty}$ and $\mathcal{D}^{\prime}$.

When $G$ is stable, then by Theorem 7.2 the variables $u$ are free over $\mathcal{S}_{+}$, so each such input there corresponds to some output $y \in\left(\mathcal{S}_{+}\right)^{p}$, and by time-autonomy there cannot exist a different causal response, so all causal responses to $u$ are in $\left(\mathcal{S}_{+}\right)^{p}$. In other words, $(u, y)$ is stable with respect to $\mathcal{S}$. The same argument establishes stability with respect to $\mathcal{S}^{\prime}$ on the condition that $G$ is weakly stable.

Conversely, suppose the input/output structure is both causal with respect to $\mathcal{C}^{\infty}$ and stable with respect to $\mathcal{S}$. This shows that $u$ is free over $\mathcal{S}_{+}$, so by Theorem 7.2 (this being the only point where we need assumptions on $\mathcal{B}_{0, y}$ or on $n$ ), $G$ is stable, as required.

Note that Theorem 7.4 effectively states that an input/output structure is both causal and stable (with respect to $\mathcal{C}^{\infty}$ and $\mathcal{S}$, respectively) if and only if the zero-input behavior $\left(\mathcal{B}^{c}\right)_{0, y}$ of the controllable part satisfies the $\mathrm{CV}$ condition, i.e., if and only if $\mathcal{B}$ has no controllable unstable poles. It is pleasing that input/output stability is determined by the poles of the system, as in the 1D case, and that the condition for input/output stability is precisely that which has been proposed for stability of the autonomous behavior $\left(\mathcal{B}^{c}\right)_{0, y}$. Also, observe that stability with respect to $\mathcal{S}$ is stronger than stability with respect to $\mathcal{S}^{\prime}$.

While we have taken time-autonomy as a prior condition for the definition of causal and therefore stable (with respect to $\mathcal{S}$ ) input/output structures, it is in fact a consequence of these two properties. For if $y \in \mathcal{B}_{0, y}$ has support in $\mathbb{R}_{+}^{n}$, then by stability (0 being a stable input!), $y \in\left(\mathcal{S}_{+}\right)^{p} \subseteq \mathcal{S}^{p}$. If $P$ is a kernel matrix representation matrix of $\mathcal{B}_{0, y}$, then it has a nonzero highest order minor $r$, and now we find that $r(\partial) y=0$, which as $y \in \mathcal{S}$ necessitates $y=0$ (e.g., by taking Fourier transforms). Thus $\mathcal{B}_{0, y}$ is time-autonomous.

As is the case for causality, Theorem 7.4 in particular implies that stability is determined by the properties of a single polynomial $d$, the least common denominator of the transfer matrix. To ascertain stability of the input/output structure with respect to $\mathcal{S}$, we need only test whether $d$ obeys the $\mathrm{CV}$ condition, i.e., whether the roots of $d$ intersect the set $\mathcal{X}^{+}$. This test amounts to checking whether a set of real algebraic equations and inequalities has a solution and so may be solved by quantifier elimination theory (e.g., [1]). An important open question is whether a simpler algorithm may be developed, making special use of the structure of $\mathcal{X}^{+}$.

We conclude with a final result which is a sufficient condition only but drops the restrictions $n \leq 2$ or $P$ a single polynomial.

Corollary 7.5. If $\mathcal{B}$ is a behavior with given input/output structure such that $\mathcal{B}_{0, y}$ is time-autonomous and $G$ is stable, then the input/output structure is causal and stable with respect to $\mathcal{S}$. This occurs in particular when $\mathcal{B}_{0, y}$ is time-autonomous and satisfies the $C V$ condition. 
Proof. The proof in Theorem 7.4 that $G$ is stable implies $(u, y)$ is causal and stable depends only on the proof $5 \Rightarrow 1$ of Theorem 7.2 , which in turn does not require the assumptions $n \leq 2$ or $P$ a single polynomial. The final claim is immediate from Lemma 2.4.

8. Conclusions. We have defined causality and stability for input/output structures with the a priori property that $\mathcal{B}_{0, y}$ be time-autonomous. This property means that any output is determined by its own past together with the input. When this property does not hold (e.g., for the heat equation), our definitions do not apply, and we believe that an entirely different approach will be necessary to define and characterize these properties in that case.

We have shown that both causality and stability are characterized by properties of the system transfer matrix or, more precisely, its least common denominator $d$. Presupposing time-autonomy, the input/output structure is causal (with respect to $\mathcal{C}^{\infty}$ ) if and only if $d$ is hyperbolic, and is stable (with respect to $\mathcal{S}$ ) if and only if $d$ satisfies the CV condition. The CV condition is precisely that which we have proposed for stability of an autonomous behavior; establishing (or disproving) that the CV condition is equivalent to stability (in a suitable sense) for an autonomous behavior is an important open question.

The obstacle to generalizing the stability results for $\mathcal{S}$ to the general $n>2$ case is in proving that the set $\mathcal{X}^{-}$has the property of being ideal-convex. This seems difficult to establish, particularly since this set is noncompact (and so it is not clear that we can approximate holomorphic functions uniformly on $\mathcal{X}^{-}$by polynomials). However, we note that $\mathcal{X}^{-}$may be bilinearly transformed into the bounded set $\left(S^{1}\right)^{n-1} \times D$, where $S^{1}$ is the unit circle and $D$ the unit disc. The stability results for $S^{1}$ require no prior assumptions but give sufficient conditions only.

Another open problem is the generalization of these results to the case of equations with real coefficients. We have used only complex coefficients here since we have applied many results from the theory of PDEs which have been developed for complex coefficients; but the real coefficient case will probably require close examination of the PDE literature.

Finally, we have developed some structure theory results for the sets $\mathcal{S}, \mathcal{S}^{\prime}, \mathcal{S}_{+}$, and $\mathcal{S}_{\oplus}^{\prime}$. The use of algebraic "local cohomology" may prove a useful approach to characterizing (Willems) closures in other situations, particularly since when we apply this tool we immediately get, as a corollary, concrete characterizations of the inclusion of one behavior in another, as in Corollaries 6.3 and 6.13. We have also been able to characterize freeness of variables over $\mathcal{S}$ for $n \leq 2$ (and over $\mathcal{C}_{0}^{\infty}, \mathcal{E}^{\prime}$ also); generalization to $n D$ depends on establishing ideal-convexity of $\imath \mathbb{R}^{n}$. The question as to whether the space $\mathcal{S}_{\oplus}^{\prime}$ is injective (and whether the space $\mathcal{S}_{+}$, to which it is dual, is flat) is also open.

\section{REFERENCES}

[1] B. F. Caviness and J. R. Johnson, Eds., Quantifier Elimination and Cylindrical Algebraic Decomposition. Texts and Monographs in Symbolic Computation, Springer-Verlag, Vienna, 1998.

[2] R. F. Curtain and H. J. Zwart, An Introduction to Infinite-Dimensional Linear Systems Theory, Texts in Applied Mathematics 21, Springer-Verlag, New York, 1995.

[3] D. Eisenbud, Commutative Algebra with a View Toward Algebraic Geometry, Graduate Texts in Mathematics 150, Springer-Verlag, New York, 1995.

[4] S. G. Gindikin and L. R. Volevich, The Cauchy problem, in Partial Differential Equations III Yu V. Egorov and M. A. Shubin (eds.), Encyclopedia of Mathematical Sciences 32, Chapter I, Springer-Verlag, Berlin, 1991. 
[5] H. Glüsing-Lürssen, Linear Delay-Differential Systems with Commensurate Delays: An Algebraic Approach, Number 1770 in Lecture Notes in Mathematics, Springer-Verlag, Berlin, 2002.

[6] L. Hörmander, On the theory of general partial differential operators, Acta Math., 94 (1955), pp. 161-248.

[7] L. Hörmander, An Introduction to Complex Analysis in Several Variables, 2nd ed., NorthHolland/American Elsevier, New York, 1973.

[8] L. Hörmander, The Analysis of Linear Partial Differential Operators I: Distribution Theory and Fourier Analysis, Springer-Verlag, Berlin, 1983.

[9] L. Hörmander, The Analysis of Linear Partial Differential Operators II: Differential Operators With Constant Coefficients, Springer-Verlag, Berlin, 1983.

[10] F. John, Partial Differential Equations, Springer-Verlag, New York, 1971.

[11] T. Y. LAM, Lectures on Modules and Rings, Graduate Texts in Mathematics 189, SpringerVerlag, New York, 1999.

[12] B. Malgrange, Division des distributions, Seminaire L. Schwartz, pp. Exposes 21-25, 1960.

[13] B. Malgrange, Systemes differentiels a coefficients constants, in Seminiare Bourbaki, Soc. Math. France, 246 (1995), pp. 79-89.

[14] E. Matlis, Divisible modules, Proc. Amer. Math. Soc., 11 (1960), pp. 385-391.

[15] S. MatsuURA, On general systems of partial differential operators with constant coefficients, J. Math. Soc. Japan, 13 (1961), pp. 94-103.

[16] M. Nacinovich, Cauchy problem for overdetermined systems, Ann. Mat. Pura Appl., 156 (1990), pp. 265-321.

[17] U. Oberst, Multidimensional constant linear systems, Acta. Appl. Math., 20 (1990), pp. 1-175.

[18] U. OBerst, Variations on the fundamental principle for linear systems of partial differential and difference equations with constant coefficients, Appl. Algebra Engrg., Comm. Comput., 6 (1995), pp. 211-243.

[19] V. P. Palamodov, Linear Differential Operators with Constant Coefficients, Springer-Verlag, New York, 1970.

[20] H. Pillai and S. Sankar, A behavioral approach to control of distributed systems, SIAM J. Control Optim., 37 (1999), pp. 388-408.

[21] H. Pillai, J. Wood, and E. Rogers, On homomorphisms of nD behaviors, IEEE Trans. Circuits Systems I. Fund. Theory Appl. (special issue on multidimensional systems), 49 (2002), pp. 732-742.

[22] J. W. Polderman and J. C. Willems, Introduction to Mathematical Systems Theory: A Behavioral Approach, Texts in Applied Mathematics 26, Springer-Verlag, New York, 1998.

[23] M. Renardy and R. C. Rogers, eds., An Introduction to Partial Differential Equation, Texts in Applied Mathematics 13, Springer-Verlag, New York, 1993.

[24] A. J. SASANE, Time-autonomy and time-controllability of $\mathcal{W}_{\mathcal{S}}$-behaviors, SIAM J. Control Optim., (2002), submitted.

[25] A. J. Sasane, E. G. F. Thomas, and J. C. Willems, Time-autonomy versus timecontrollability, Systems Control Lett., 45 (2002), pp. 145-153.

[26] A. J. SASAne, On the Willems closure with respect to $\mathcal{W}_{\mathcal{S}}$, IMA J. Math. Inform. Control, 20 (2003), pp. 217-232.

[27] A. J. SASAne, Time-autonomy and time-controllability of 2-D behaviors that are tempered in the spatial direction, Multidimens. Systems Signal Process., 15 (2004), pp. 97-116.

[28] S. Shankar, An obstruction to the simultaneous stabilization of two n-D plants, Acta Appl. Math., 36 (1994), pp. 289-301.

[29] S. Shankar, The Nullstellensatz for systems of PDE, Adv. Appl. Math., 23 (1999), pp. 360374.

[30] S. Shankar. Can one control the vibrations of a drum? Multidimens. Systems Signal Process., 11 (2000), pp. 67-81.

[31] S. Shankar, Geometric completeness of distribution spaces, Acta Appl. Math., 77 (2003), pp. $163-180$.

[32] S. Shankar And V. R. Sule, Algebraic geometric aspects of feedback stabilization. SIAM J. Control Optim., 30 (1992), pp. 11-30.

[33] M. E. VAlChER, Characteristic cones and stability of two-dimensional autonomous behaviors, IEEE Trans. Circuits Systems I Fund. Theory Appl., 47 (2000), pp. 290-294.

[34] J. C. Willems, Paradigms and puzzles in the theory of dynamical systems, IEEE Trans. Automat. Control, 36 (1991), pp. 259-294.

[35] J. Wood, Modules and behaviors in nD systems theory, Multidimens. Systems Signal Process., 11 (2000), pp. 11-48. 
[36] J. Wood, Key problems in the extension of module-behavior duality, Linear Algebra Appl., 351/352 (2002), pp. 761-798.

[37] J. Wood, U. Oberst, E. Rogers, And D. H. Owens, A behavioral approach to the pole structure of one-dimensional and multidimensional linear systems, SIAM J. Control Optim., 38 (2000), pp. 627-661.

[38] S. ZAMPIERI, Causal input/output representation of $2 D$ systems in the behavioral approach, SIAM J. Control Optim., 36 (1998), pp. 1133-1146.

[39] P. Zaris, J. Wood, AND E. Rogers, Controllable and uncontrollable poles and zeros of $n D$ systems, Math. Control Signals Systems, 14 (2001), pp. 281-298.

[40] E. ZERZ, Extension modules in behavioral linear systems theory, Multidimens. Systems Signal Process., 12 (2001), pp. 309-327. 